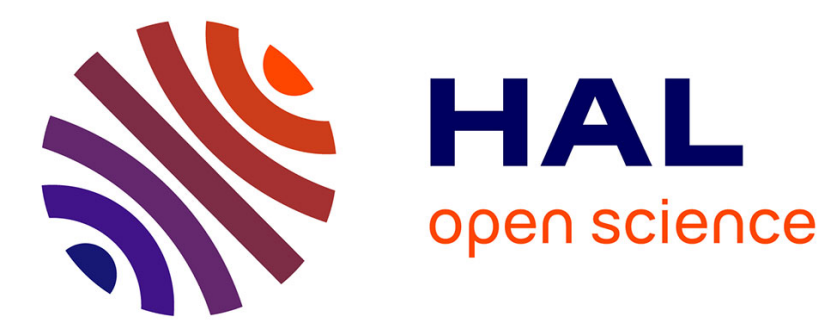

\title{
Simultaneous Pharmacokinetic-Pharmacodynamic modeling
}

\author{
Pierre-Louis Toutain
}

\section{To cite this version:}

Pierre-Louis Toutain. Simultaneous Pharmacokinetic-Pharmacodynamic modeling. Comparative Pharmacokinetics: Principles, Techniques, and Applications, 2nd Edition, John Wiley and Sons, pp.255-294, 2011, 978-0-8138-2993-7. hal-01137047

\section{HAL Id: hal-01137047 \\ https://hal.science/hal-01137047}

Submitted on 8 Mar 2017

HAL is a multi-disciplinary open access archive for the deposit and dissemination of scientific research documents, whether they are published or not. The documents may come from teaching and research institutions in France or abroad, or from public or private research centers.
L'archive ouverte pluridisciplinaire HAL, est destinée au dépôt et à la diffusion de documents scientifiques de niveau recherche, publiés ou non, émanant des établissements d'enseignement et de recherche français ou étrangers, des laboratoires publics ou privés. 


\title{
13 Simultaneous Pharmacokinetic- Pharmacodynamic Modeling
}

\author{
with Pierre-Louis Toutain
}

The initial chapter of this text covered the pharmaceutical and pharmacokinetic phases of drug disposition encompassed in quantifying absorption, distribution, metabolism, and elimination (ADME). The final phase of modeling involves the coupling of drug concentration at the active site (pharmacokinetics, PK) to its biological (pharmacodynamics, PD) and its clinical effects (therapeutics). The present chapter will introduce the objectives, underlying concepts, methods, information, advantages as well as the limitations of the quantitative approaches to link these processes into complete models of drug disposition and action; an approach referred to as PK/PD modeling. Emphasis in this chapter will be placed on the major modeling steps for the PD components of the model. The PK approaches have been extensively discussed in Chapters 8-10. The application of PK/PD modeling to rational development of veterinary drugs will be stressed. In many ways, the material in this chapter could actually comprise a separate book focused on what could be referred to as quantitative pharmacology or pharmacometrics. As this complex area is developed, the reader will see numerous parallels to the development of pharmacokinetic models discussed throughout the text. In order to be as complete as possible, this chapter also includes a number of recent applications specifically relevant to veterinary medicine.

\subsection{OVERVIEW ON PK/PD MODELING}

PK/PD modeling consists of describing and explaining the time course of the drug effect (intensity or frequency of the observed response as quantified by PD) via the time course of its concentration in the plasma (PK). The primary objective of PK/PD modeling is to estimate, in vivo, key PD parameters of a drug to predict the time course of a drug effect under physiological and pathological conditions. Another objective of a PK/PD model is to increase the understanding of the underlying mechanisms of drug action.

$\mathrm{PK} / \mathrm{PD}$ modeling is a versatile tool which is mainly used in veterinary medicine to select rational dosage regimens (dose, dosing interval) for confirmatory clinical testing. The ultimate goal in therapeutics is to control a drug response, not drug exposure. In a clinical setting, this consists of the physician applying feedback (e.g., clinical outcome) from previous drug administration to directly adjust a dosage regimen as explained in Chapter 12. This approach is efficient for some drugs such as anesthetics or opioids for which the effects

Comparative Pharmacokinetics: Principles, Techniques, and Applications, Second Edition. Jim E. Riviere.

(C) 2011 Jim E. Riviere. Published 2011 by John Wiley \& Sons, Inc. 
are immediately observable and need to be adjusted without delay. However, for most drugs, this experience-based approach remains rather inefficient. For instance, optimizing the effect of an antibiotic in terms of efficacy (e.g., minimum inhibitory concentration [MIC]) or resistance through clinical observation of symptoms is not sufficient and the recourse to more advanced approaches is required. One should realize that systemic drug effect is mediated via the time-course profile of drug concentration at the site of actionreferred to as the biophase-and for drugs acting systemically, biophase drug exposure bears a proportional relationship with plasma concentration. Thus, monitoring plasma concentrations is a way to indirectly control the response to a drug, which is the essence of the PK/PD approach, that is, considering plasma concentration as the turntable between a dosage regimen and a clinical response. This implies that PK and PD events have to be considered simultaneously and linked using a fit for the purpose model that is a PK/PD model.

\subsubsection{When and why PK/PD modeling should be used}

The efficiency of drug development can be greatly improved through the use of a PK/PD modeling approach, rather than by studying in parallel PK and PD data. PK/PD modeling is applicable to all phases of drug development. PK/PD can be used very early in drug development from drug target discovery to bridging in vitro drug action and in vivo drug effects as is the case for antibiotics, or to discard compounds having an initial attractive in vitro potency but which are unable to maintain an appropriate sustained in vivo exposure. Later in preclinical drug development, the primary objective of PK/PD analysis is to provide estimates of the key in vivo PD properties of a drug (i.e., its potency, efficacy, and selectivity) and to begin to explore the question of a dosage regimen. In veterinary medicine, the PK/PD modeling approach also offers a conceptual framework for extrapolation between species and to predict drug effects under different physiological or pathophysiological conditions. Later during clinical development, modeling clinical responses taking into account not only drug effects but also disease progression or any relevant physiopathological events on which the drug is acting or interacting may help to finalize an optimal dosage regimen.

By separating the two main sources (PK and PD) of inter- and intraindividual variability, the PK/PD modeling approach can be used during clinical trials to identify the key factors allowing a dosage regimen to be individualized, taking into account either PK- or PDrelevant covariables (population PK/PD modeling, see Chapter 16). Finally, PK/PD modeling can be used prospectively for some clinical trial simulations (CTS) to streamline their design or to explore "what-if" scenarios. Fig. 13.1 summarizes how PK/PD concepts can be used in preclinical and clinical product development in veterinary medicine.

\subsubsection{Dosage regimen determination: from dose titration to PK/PD modeling}

In veterinary medicine, the selection of a dose is generally based on dose-ranging studies using a simple parallel design. With parallel design, animals (healthy, experimentally infected, etc.) are randomly allocated to several dose levels and the effects are compared using a standard statistical test of the hypothesis as shown in Fig. 13.2. This design has two serious limitations: first, it is unable to provide information on the shape of the individual dose-response relationship, which is relevant to discuss the drug selectivity that is sorting out desired and undesired drug effeets. Second, the dose designated as the "effective 


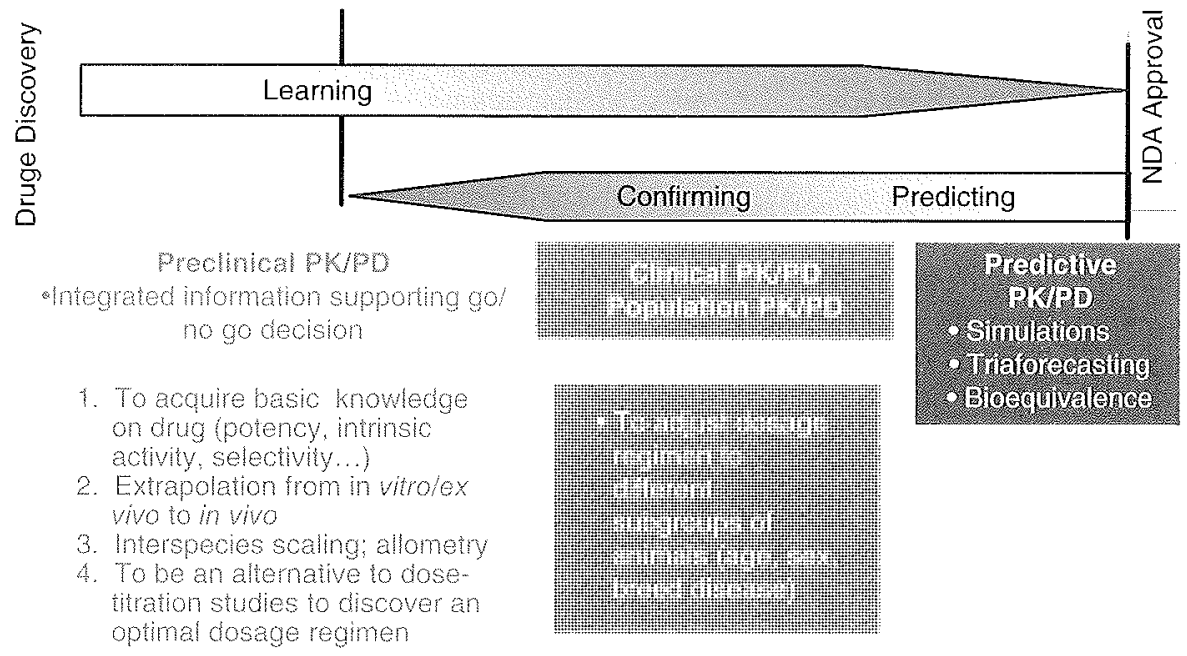

Fig. 13.1 Potential applications of PK/PD concepts during preclinical and clinical development of a veterinary product. Adapted from Meibohm and Derendorf (2002). NDA, new drug application.

Parallel design

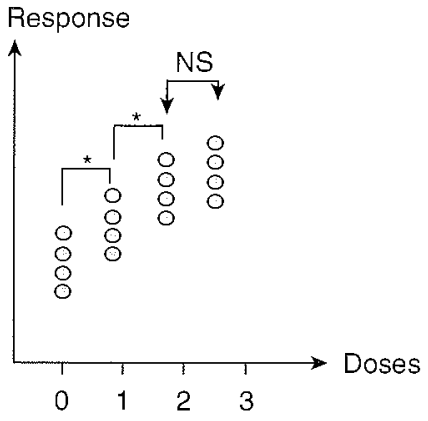

Data analysis:

Results:

Conclusion
Analysis of variance

$\mathrm{D} 0>\mathrm{D} 1>\mathrm{D} 2=\mathrm{D} 3$

$\mathrm{D} 2$ is selected
Crossover design

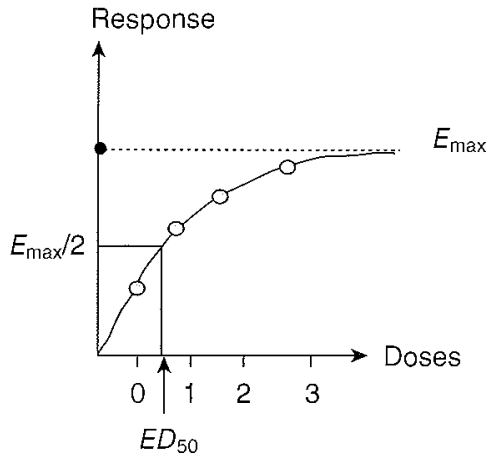

PD modeling

$E_{\max }$ and $E D_{50}$ are estimated Any dose can be selected

Fig. 13.2 Parallel (left) versus crossover (right) design for a dose-titration study. In a parallel design, animals (here $n=4$ per group) are randomly assigned to one of the tested doses $(0,1,2$, or 3$)$. Data analysis is performed by a test of the hypothesis (analysis of variance, ANOVA), the selected dose being one of the tested doses (no interpolation). Here, D2 would be selected because it gives a significantly higher response ( $\left(^{*}\right)$ than D1 but is not significantly (NS) different from D3. In a crossover design, all animals receive every dose to be tested and individual dose-effect curves are generated. For each individual curve, $P D$ parameters $\left(E_{\max }, E D_{50}\right)$ can be computed and any dose over the tested range can be selected. Crossover but not parallel designs provide information about the shape of the dose-response relationship and the variability within the population. 
dose" is not obligatorily the optimal dose. Indeed, the selected dose is highly dependent on the power of the design (number of tested animals), and trials with a small sample size generally lead to the selection of an oversized dose.

This classical parallel design remains the only usable one for antiparasitic and antibiotic drugs, because irreversible drug effects (i.e., pathogen eradication) are the pivotal outcomes. In contrast, for drugs acting reversibly on a physiological system (e.g., cardiovascular, nervous system, kidney), the most attractive design consists of using the so-called crossover design where each animal is tested with the different doses being investigated. In this way, individual dose-response curves can be generated. For a given animal, the dose-effect relationship can be described by a basic relationship of the form:

$$
\text { Effect }=E_{0} \pm \frac{E_{\max } \cdot D_{0 s e^{n}}}{E D_{50}^{n}+D_{0 s e^{n}}}
$$

where Effect (dependent variable) is the predicted effect for a given Dose (independent variable); $E_{0}$ is the effect without the drug (i.e., a placebo effect or the baseline response of the system); $E_{\text {nax }}$ is the maximum possible effect and $E D_{50}$ is the dose producing half $E_{\max } . E_{\max }$ is a measure of the drug efficacy, whereas $E D_{50}$ is a measure of the drug potency. $E_{0}, E_{\max }$, and $E D_{50}$ are parameters. When an exponent $(n)$ is included in the model, it reflects the slope of the dose-effect relationship and can provide information on drug selectivity for the tested effect; this equation will be fully presented and discussed later with the presentation of Equation 13.3. However, at this point, the reader should note the similarity of this nonlinear equations to those encountered in Chapter 5 for protein binding (Eq. 5.4) and in Chapter 10 (Eq. 10.4) for drug biotransformation.

When considering Equation 13.1, it should be noted that $E D_{50}$ is not a genuine PD parameter but rather a hybrid $\mathrm{PK} / \mathrm{PD}$ variable. Actually, $E D_{50}$ incorporates three different separate determinants, as described by the following relationship:

$$
E D_{50}=\frac{\text { Clearance } \cdot E C_{50}}{\text { Bioavailability }}
$$

where $E D_{50}$ is a daily dose able to maintain an average plasma concentration equal to $E C_{50}$; $E C_{50}$ is the steady-state plasma concentration producing half $E_{\max }$; Clearance is the daily plasma clearance $\left(\mathrm{Cl}_{\mathrm{B}}\right.$ from earlier chapters), and Bioavailability is the extent of the systemic bioavailability (for the extravascular route of drug administration $-F$ ) as previously defined in Chapter 8.

Fig. 13.3 shows the fundamental differences between a dose-ranging trial (also called dose-titration trial) and a PK/PD trial. Fig. 13.4 further illustrates this difference in a study designed to determine a gonadotropin-releasing hormone $(\mathrm{GnRH})$ dose to reestablish estrous cycles in cows with ovarian cysts, using the pituitary luteinizing hormone (LH) response as a surrogate end point.

Both approaches aim at documenting the same relationship between dose and drug response. In a PK/PD analysis, the dose is replaced by the plasma concentration profile (or the area under the curve [AUC], i.e., the internal exposure) to explain the effect closer to the site of action independent of confounding PK factors acting on the administered dose. It enables one to estimate the drug potency in terms of an $E C_{50}$ (or $\mathrm{AUC}_{50}$ ) rather than $E D_{50}$. Unlike $E D_{50}, E C_{50}$ is a genuine PD parameter. For a given end point, a single (steady- 


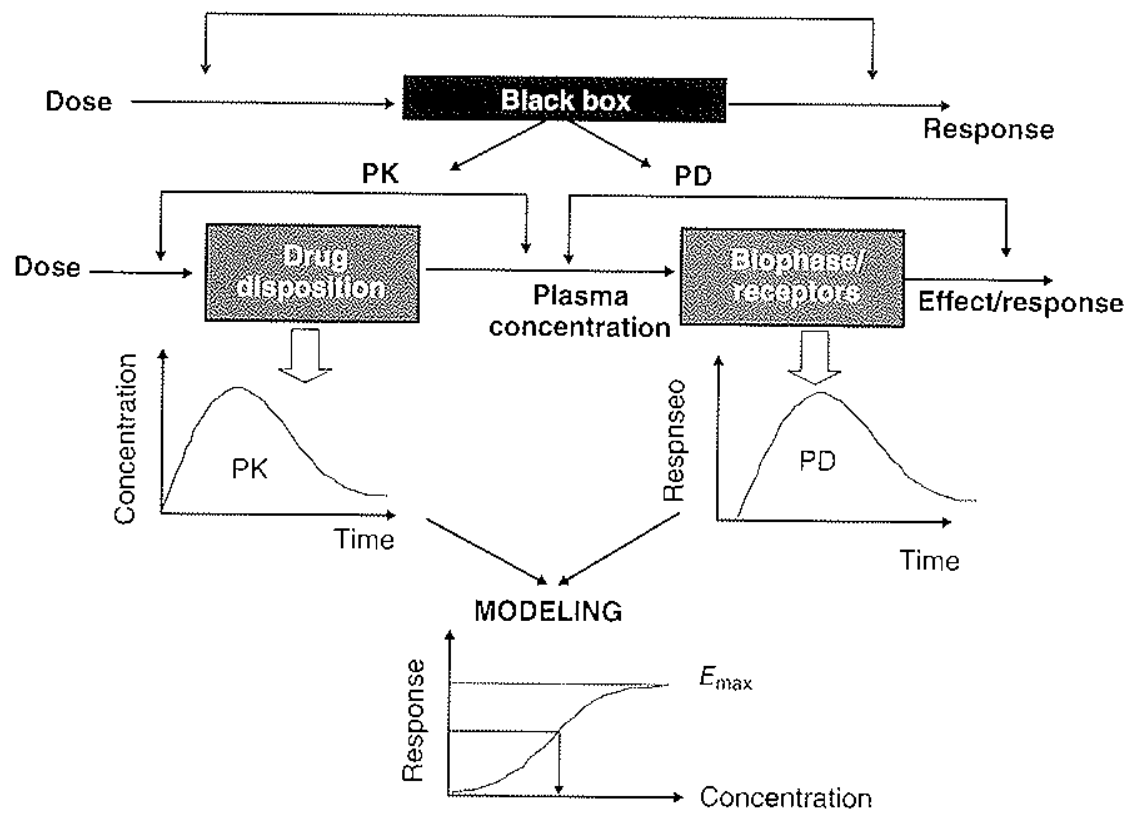

Fig. 13.3 Dose-effect relationship versus PK/PD modeling. Both approaches dim at documenting the some relationship between dose and diug response. A dose-effect relationship is a block-box opprooch in which the dose is the explicative variable of drug response. In o PK/PD approach, the black box is opened, thereby enabling the two primary processes that separate dose from response to be recognized. In the first step (PK), the dose is transformed into a plasma concentration profile. In the second step (PD), the plasma concentration profile becomes the voriable that explains the diug response. The difficulty with the PK/PD approach is that the development of the effect and plasma concentrations over time is usually not in phase. This means that a hysteresis laap is observed when the response is plotted against plasma concentrations and data modeling is required to estimate the PD parameters $\left(E_{\text {max }}, E C_{50}\right.$, and slope).

state) $E C_{50}$ value exists, which is influenced by neither $\mathrm{PK}$ parameters nor the route of administration or the formulation. Moreover, because $E C_{50}$ is a drug-dependent parameter (ED 50 is both a drug- and formulation-dependent variable), the use of a $\mathrm{PK} / \mathrm{PD}$ approach precludes the need for multiple trials as with dose-ranging. If a drug sponsor decides to develop another formulation, he will not need to perform a new PK/PD trial, but simply at new PK study to predict the infuence of the bioavalability factor on the effect. For this reason, $E C_{50}$ is much more attactive than $E D_{50}$, and its detemination is one of the main goals of a $\mathrm{PK} / \mathrm{PD}$ experiment.

\subsubsection{Plasma concentration as a "driving force" for pharmaco/toxicodynamic effects}

As discussed in Chapters 2 and 5 , it is a tenet in pharmacology that the free plasma concentration is the driving concentration "controlling" all other local concentrations after a systemic drug administration. In addition, plasma remains the most easily accessible body compartment to measure free drug concentrations. Thus, the plasma concentration can play the genuine role of a "driving force" for a pharmacodynamic or a toxicodynamic effect, consistent with it being the central or reference compartment in both compartmental and 

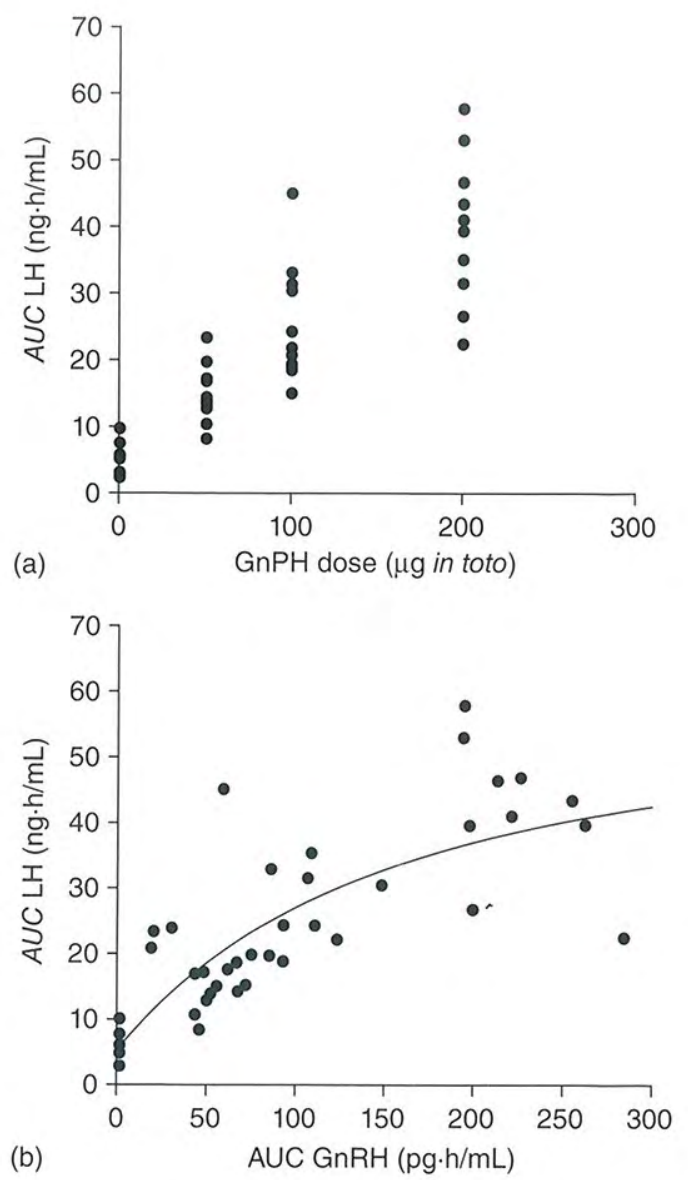

Fig. 13.4 Dose versus exposure-effect relationship. (a) The classical dose-effect relationship is plotted with the gonadotropin-releasing hormone $(\mathrm{GnRH})$ dose $(0,50,100$, or $200 \mu \mathrm{g}$ in toto) as the independent variable and the overall $\mathrm{LH}$ response (AUC LH, nanogram·hours/milliliter). When analyzed as a parallel design (i.e., ignoring that each cow was tested four times), this design is unable to provide information on the shape of the individual dose-response curves and the effective dose is imposed by the statistical analysis, that is, by testing the null hypothesis with an analysis of variance (ANOVA). (b) The dose, as an explicative variable, has been replaced by the individual GnRH exposure (GnRH AUC, picogram hours) milliliter), which allows better characterization of the mean response curve; using a minimal PK/PD model, GnRH efficacy and potency can be computed. This approach is a "naive pooled data approach" and does not guarantee that the individual concentration-effect relationship has a similar shape to the mean shape, and a more advanced PK/PD modeling consisted of analyzing the individual time development of LH responses versus the plasma concentration versus time of GnRH (see Fig. 13.19).

stochastic PK models developed in Chapters 8 and 9. The plasma concentration profile is inherently more informative than the administered dose and in most cases the in vivo plasma concentration of a drug has proved to be the best predictor of the drug's effect. The dose is only a nominal mass fixed by the clinician, providing no intrinsic biological information. In contrast, the concentration versus time profile is controlled by both the dose (clinician) and the animal (through its physiological processes such as plasma clearance and distribution). In addition, plasma concentration profiles provide temporal data, allow- 
ing a $\mathrm{PK} / \mathrm{PD}$ trial to include time as an independent variable in a $\mathrm{PK} / \mathrm{PD}$ model, enabling, for example, not only a dose, but also a dosage interval to be determined.

\subsubsection{Tissue sites other than plasma concentrations to explain effects}

Any biological flud concentrations having the status of "driving force" can be considered for PK/PD modeling if the concentrations are kinetically and biologically closer to the effect. For example, all kidney loop diuretics act on the apical membrane transport of $\mathrm{Na}^{+}$ and $\mathrm{Cl}^{-}$of the thick ascending loop of Henle in the kidney by inhibiting $\mathrm{Na}^{+}-\mathrm{K}^{+}-\mathrm{Cl}^{-1}$ cotransport processes that is from the urine space, not from the blood compartment. This location can be appreciated by examining Fig. 6.4 in Chapter 6 . This requires that loop diuretics should gain access to the urine (filtration, secretion) to locally achieve high enough concentrations. From a PK/PD modeling perspective, the urine concentration is the relevant driving concentration to describe the diuretic effects of loop diuretics and to investigate the consequences of impaired renal function. In contrast, plasma concentration is the relevant concentration to investigate systemic effects of these agents on $\mathrm{Na}^{+}-\mathrm{K}^{+}{ }^{+} \mathrm{Cl}^{-}$cotransport processes in nonrenal tissue and especially to investigate any side effects of loop diuretics such as deafness.

Transudate and exudate fluids inside a tissue cage or indwelling microdialysis probe can also be selected as a driving force to explain the effect of antibiotics on microorganism or the effect of nonsteroidal anti-inflammatory drugs (NSAIDs) on targeted enzyme systems. Other fuids have been used to establish a mechanistic PK/PD relationship including milk (for antibiotics), synovial fuid for NSAIDs, as well as cerebrospinal fuid for anesthetics and analgesics. Yet, plasma concentration remains the most useful biological matrix for monitoring plrposes, the ultimate objective being to control this plasma concentration by an appropriate dosage regimen.

\subsection{THE BUILDING OF PK/PD MODELS}

\subsubsection{Components}

The process for building a structural PK/PD model generally involves four different submodels:

- a PK model transfoming the dose into a concentrations versus time profile. These have been presented in Chapters 8-10;

- a link model describing drug transfer from plasma to the biophase;

- a system model that describes the physiological system or the pathological process on which the drug is acting;

- a PD model relating biophase concentration to an effect on the system.

In addition, any structural PK/PD model can be completed by a statistical model that describes the error component of the model and that is typically estimated in population PK/PD investigations, as presented in Chapter 16. Depending on the drug and the purpose of the modeling, the four structural submodels may or may not be involved. We will first describe the different PD models. 


\subsubsection{Pharmacodynamic models}

There are two main types of PD models, describing either a graded concentration-effect relationship or a quantal concentration-response relationship.

A graded model is used when the response to different drug concentrations can be quantified on a scale (e.g., body temperature, survival time). In a quantal model (also known as a fixed-effect model or all-or-none effect model), the described effects are nominal (categorical) (e.g., dead or alive, parasitic cure or not, appearance of unwanted effects or not). A graded dose-effect relationship can be measured on a single animal that is exposed to a range of doses, the intensity of the response being related to the dose. For quantal dose-response (or exposure-response) relationships, the dose or exposure is not related to the intensity of the effect but to the frequency of an all-or-none effect. Thus, quantal doseresponse relationships are established in a population of animals that are exposed to a range of doses. Quantal responses are often clinical end points, whereas graded responses are often biomarker concentrations or surrogates used to replace clinical end points of ultimate interest in PK/PD models.

The most general model for a graded effect relationship is the Hill model, also known as the sigmoidal $E_{\max }$ model:

$$
E(t)=E_{0}+\frac{E_{\max } \cdot C^{n}(t)}{E C_{50}^{n}+C^{n}(t)}
$$

where $E(t)$ is the effect observed for a given concentration at time $t$, that is, $C(t)$. $C(t)$ describes the PK model and explicitly introduces the variable time $(t)$ in the model (that is the main difference with Eq. 13.1); $E_{\max }$ is the maximal effect attributable to the drug; $E C_{50}$ is the plasma concentration producing $50 \%$ of $E_{\max }$; and $n$ is the Hill coefficient (no dimension), representing the slope of the concentration-effect relationship. The power function $\left(C^{n}\right)$ first encountered in Chapter 8 (see Eq. 8.59) has been introduced in this equation to bring an appropriate flexibility for describing a great variety of shapes of the concentration-effect relationships seen in Fig. 13.5. Equation 13.3 was first formulated in 1910 for studying the $\mathrm{O}_{2}$ and hemoglobin association-dissociation process and subsequently used for more complex binding processes such as drug-receptor binding. When $n=1$, Equation 13.3 is analogous to the Michaelis-Menten equation as derived and explained in Chapter 10, Equation 10.4. Many drug effects involve modulation of a physiological variable (e.g., blood pressure) and the inclusion of the term $E_{0}$ in Equation 13.3 indicates the presence of a baseline effect. $E_{0}$ can also represent a placebo effect. $E_{0}$ may not be a constant: if the baseline is subjected to some variation, it should be modeled (see later).

The sigmoidal model shows a great degree of flexibility and other simpler models can be viewed as particular cases of the sigmoidal model. For example, when $n=1$, the Hill model is reduced to the classical $E_{\max }$ model, which corresponds to a rectangular hyperbolic function:

$$
E(t)=E_{0} \pm \frac{E_{\max } \cdot C(t)}{E C_{50}+C(t)}
$$

The $E_{\max }$ model originates from the classical receptor theory, but in PK/PD modeling, it serves primarily as an empirical model. From this equation, the three main PD parameters can be defined (Figs. 13.6 and 13.7). 


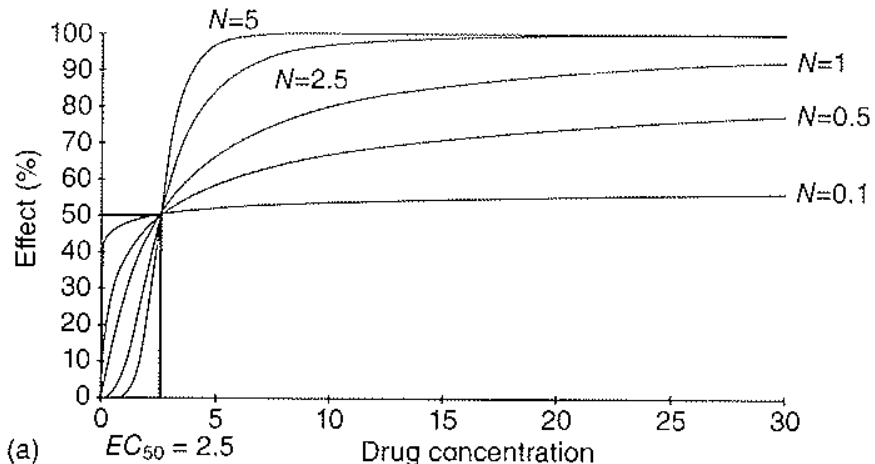

(a)

Drug cancentration

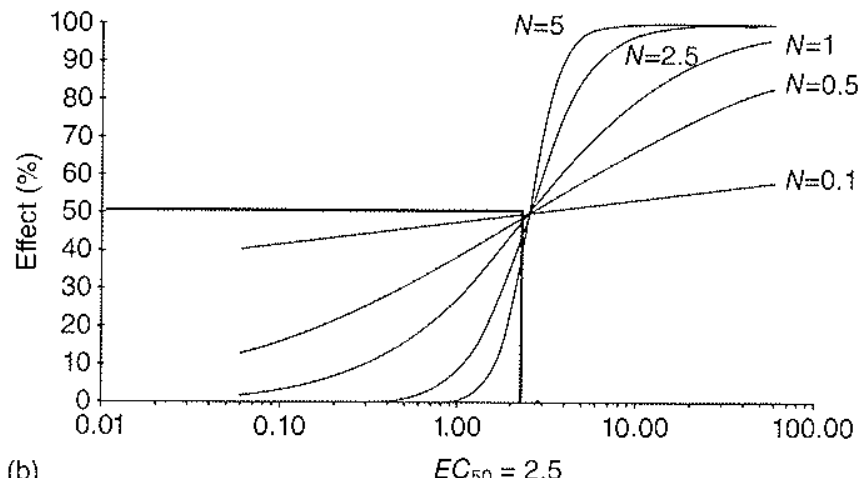

(b)

$$
E C_{50}=2.5
$$

Fig. 13.5 Hill model. Influence of the Hill coefficient $(N)$ on the shape of the concentration-effect relationship; simulation of the effect change (\%) for $N=0.1,0.5,1,2.5$, and 5. (o) Arithmetic scale. (b) Logorithmic scale.

If $n=1$ and if $C(t)$ is much lower than $E C_{50}$, then the sigmoidal model collapses to the simple linear model of Fig. 13.8:

$$
E(t)=E_{0}+\frac{E_{\mathrm{max}}}{E C_{50}} \cdot C(t)
$$

with $E_{n a x} / E C_{50}$ as a slope. This can be rewritten more simply as:

$$
E(t)=E_{0}+\text { Slope } \cdot C(t)
$$

Note that this equation is writen in the now all-too familiar slope-intercept fom $(y=a+b x)$ whose solution is similar to that presented in Chapter 8 for solving PK equations. A negative slope can be used if the drug has an inhibitory effect. Although this linear PD model is simple and easy to use, it is unsuitable for accurately predicting the baseline effect at time 0 (i.e., $E_{0}$ ).

If the tested $C(t)$ are of the same order of magnitude as $E C_{50}$, it is often observed that a simple log-linear model is sufficient to describe drug action:

$$
E(t)=E_{0}+S \operatorname{lope} \cdot \log C(t)
$$



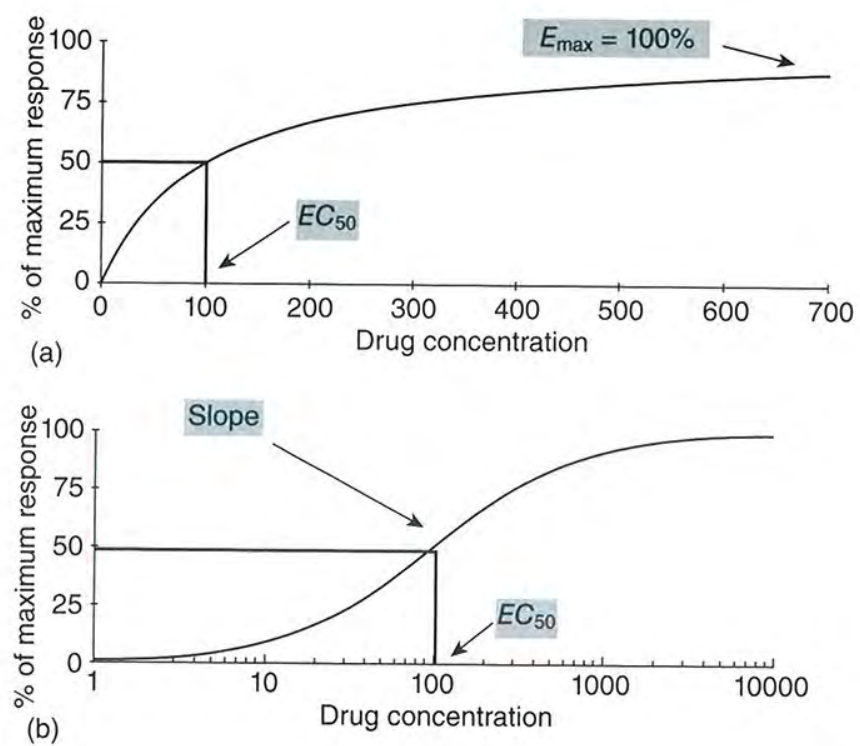

Fig. 13.6 $E_{\max }$ model plotted with arithmetic (a) and semilogarithmic scale (b). (a) When the drug response (arithmetic $y$-axis) is plotted against the tested dose (arithmetic $x$-axis), a hyperbolic relationship is offen observed with a maximal effect noted $E_{\max } . E C_{50}$ is the concentration that produces an effect equal to half $E_{\text {mox. }}$ (b) When the same data are represented using a log-X scale, the relationship becomes sigmoidal with a more or less steep slope. The advantage is to compress the concentration scale and to visualize the slope more easily. A sigmoidal curve allows the definition of three drug parameters, namely $E_{\max } E C_{50}$, and the slope $(\Delta Y / \Delta X)$. $E_{\max }$ describes the drug efficacy and $E C_{50}$ describes the drug potency. The slope (measured by the $n$ of the Hill equation-see Eq. 13.3 in the text) indicates the sensitivity of the dose-effect curve. The slope of the curve is involved in the drug selectivity (see Fig. 13.13).

where the Slope parameter can be computed by linear regression. In in vitro steady-state conditions, $C(t)$ is a constant $(C)$ and Equation 13.7 has been used extensively to characterize basic in vitro PD drug properties such as drug potency and to test competitive versus noncompetitive antagonism between drugs. However, it should be realized that Equation 13.7 describes only a limited range of the concentration-effect relationship, namely between about $20 \%$ and $80 \%$ of the possible $E_{\max }$ as seen in Fig. 13.9. Precautions must be taken when using this model beyond this range (possible overestimation of drug effect at over $80 \%$ of $E_{\max }$ and underestimation below $20 \%$ of $E_{\max }$ ) and the availability of computer software to fit by nonlinear regression data to the $E_{\max }$ or the Hill model no longer justify the use of Equation 13.7.

When the drug effect corresponds to the inhibition of a biological process, the drug effect is subtracted from the baseline $\left(E_{0}\right)$ and Equation 13.3 can be rewritten as:

$$
E(t)=E_{0}-\frac{I_{\max } \cdot C^{n}(t)}{I C_{50}^{n}+C^{n}(t)}
$$

where $I C_{50}$ is the concentration producing $50 \%$ of the maximum inhibition effect $\left(I_{\max }\right)$. If the drug is capable of reducing the baseline effect to zero, that is, $I_{\max }$ is equal to $E_{0}$, then Equation 13.8 can be simplified to the so-called fractional $I_{\max }$ model: 

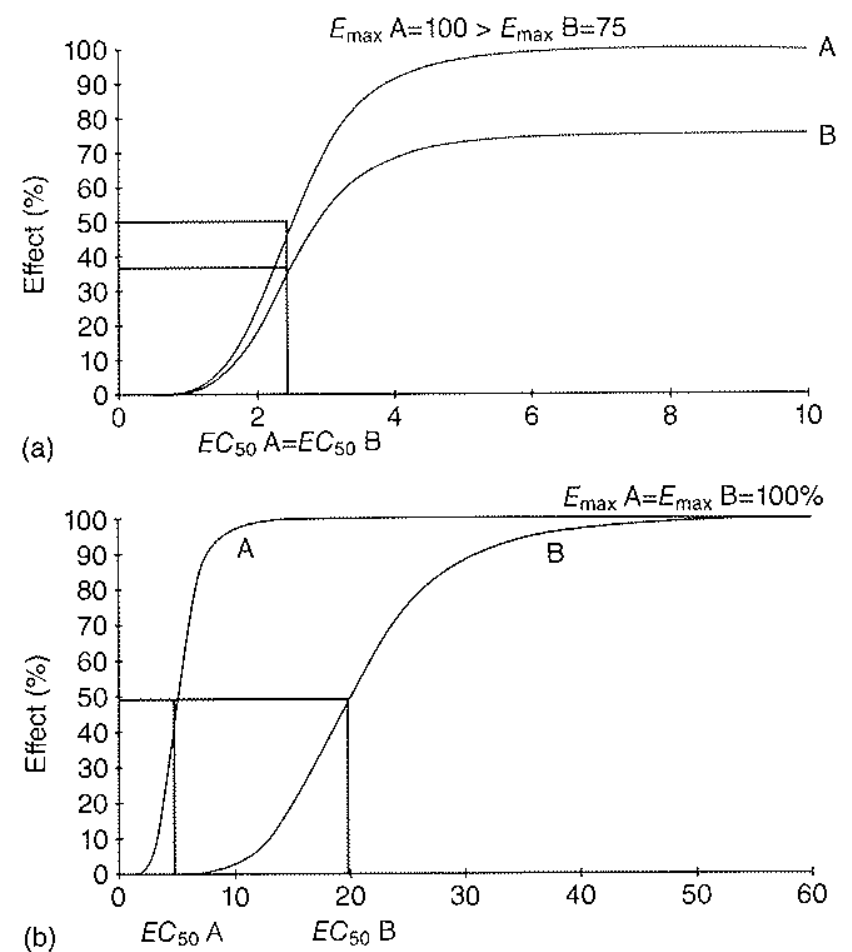

(b)

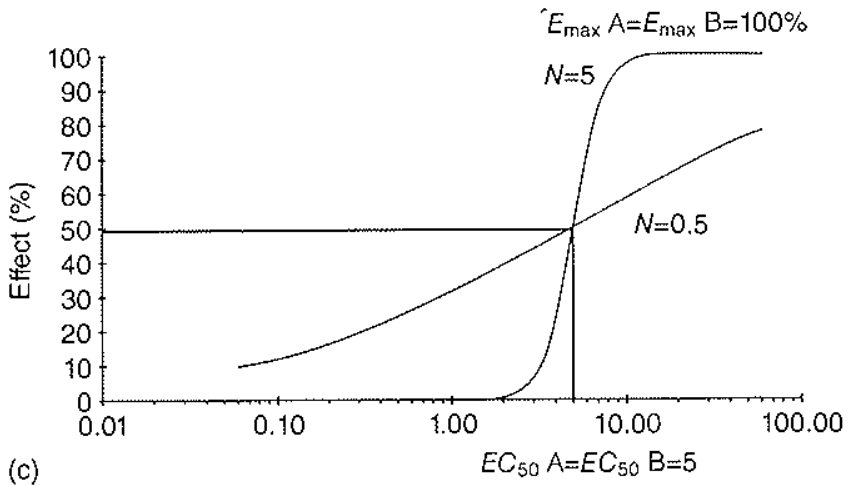

Fig. 13.7 Dose-response relationship and characterization of the three main pharmacodynamic parameters. (a) Efficocy. Drug $A$ is more efficacious than drug 8 because $E_{\max } A=100$ is higher thon $E_{\text {r:ax }} B=75$, whereas drugs $A$ and 8 have the same potency (same $E C_{50}$ ). (b) Potency. Drug $A$ is said to be more potent than drug $B$ because $E C_{50} A$ is lower than $E C_{50} B$, but drugs $A$ and $B$ have the some efficacy (same $E_{\text {mox }}$ ). (c) Sensitivity. The sensitivity of drug $A$ is higher than the sensitivity of drug $B$ because the slope of the dose-cancentration relotionship is steeper far drug A (Hill coefficient of 5) than far drug $B$ ( $H$ ill coefficient of 0.1 ), whereas they have the same potency (same $E C_{50}$ ) and efficacy (same $E_{\text {mitax }}$ not shown). 


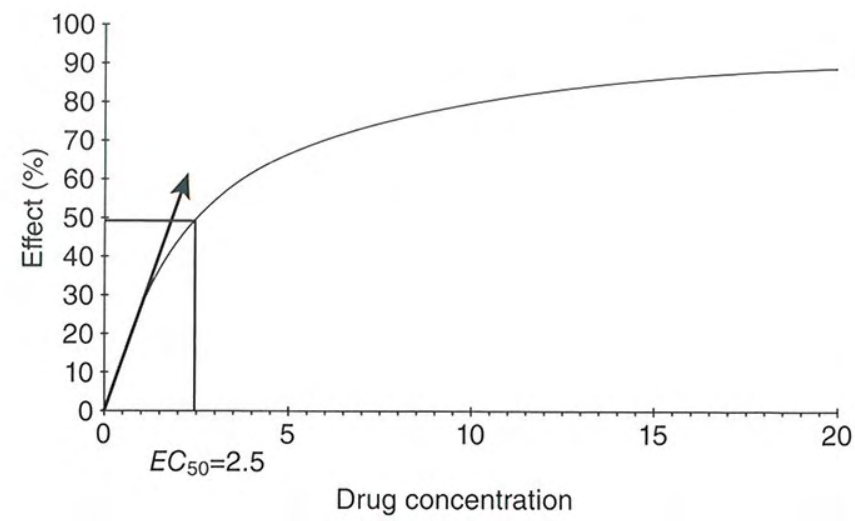

Fig. 13.8 Linear model: if the effect is measurable in the initial portion of an $E_{\max }$ model the concentration-effect relationship appears to be linear between about $0 \%$ and $20-30 \%$ of $E_{\max }$.

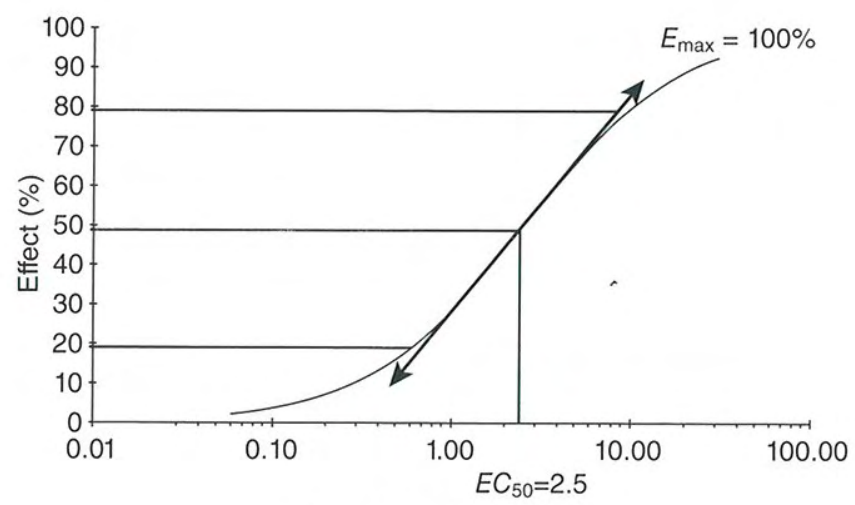

Log-drug concentration

Fig. 13.9 Log-linear model: if the effect is measured around the $E C_{50}$ of an $E_{\operatorname{mox}}$ model, the concentrationeffect relationship appears to be linear on a log-scale over a range of plasma concentrations ranging typically from $20 \%$ to $80 \%$ of $E_{\max }$.

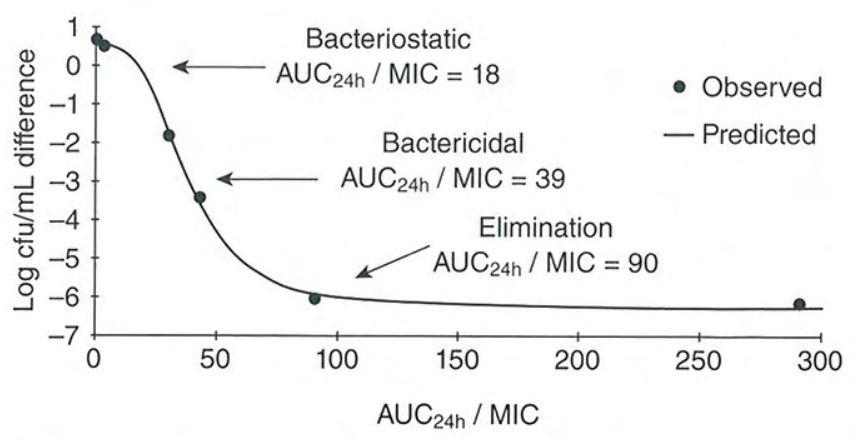

Fig. 13.10 Example of an inhibitory sigmoidal $E_{\operatorname{mox}}$ model establishing a relationship for bacterial count versus ex vivo $\mathrm{AUC}_{24 \mathrm{~h}} / \mathrm{MIC}$ in a goat for a pathogenic strain of $\mathrm{M}$. haemolytica. It illustrates what AUC/MIC values are required for bacteriostatic and bactericidal effects and eradication of bacteria (from Aliabadi and Lees, 2001). 


$$
E(t)=E_{0(0)}\left(1-\frac{C(t)^{n}}{I C_{30}^{n}+C(t)^{n}}\right)
$$

More sophisticated $E_{\text {max }} / I_{\text {mix }}$ models can be developed to investigate combined drug action as is the case for many NSAIDs that are used as racemates in veterinary medicine. For example, a PD model including both $S$ - and $R$-ketoprofen plasma concentrations was used to describe the analgesic effect of ketoprofen in piglets by Fosse et al. (2010). It was assumed that the two enantiomers produced their analgesic effect by two separate mechanisms of action and a simple additive $I_{\max }$ model was selected:

$$
\text { Effect }_{R+S}=H \text { yperalgesia(t) }\left[1-\left(\frac{C_{p r}}{I C_{50 r}+C_{p r}}+\frac{C_{p s}}{I C_{50 s}+C_{p s}}\right)\right]
$$

where Hyperalgesia( $(t)$ is a function describing the time-dependent hyperalgesia due to a paw kaolin administration (actually described by Eq. 13.19). $C_{p s}$ and $C_{p r}$ are plasma concentration of the $S$ - and $R$-ketoprofen, respectively, $I C_{50 r}$ and $I C_{50 \text { s }}$ are the $R$ - and $S$-ketoprofen plasma concentrations, which are capable, when acting alone, of producing half the maximum inhibitory effect on Hyperalgesia(t). Jonker et al. (2005) presented a series of PD models for combined drug action (multiple ligands, additive vs. competitive effects, stereoisomers, multiple receptors, and cascade activity).

Equations 13.3-13.10 contain several parameters $\left(E_{0}, E_{\text {nax }}, E C_{50}\right.$ or $/ C_{50}$, and $\left.n\right)$. The ultimate goal of PK/PD modeling is to estimate both the mean and variance of these parameters from $E(t)$ observations obtained over a range of $C(t)$ values.

When an all-or-none effect is observed due to the drug's mechanism of action (antiepileptic drug, antiarthythmic drug, etc.), or when the selected end point is binary (cured or not cured for parasiticides), the concentration-response curve represents the frequency at which a concentration of a drug produces the all-or-none effect. For this kind of end point, data with exactly two possible outcomes, quantal or percent-concentration relationship (fixed-effect model), are in order: The link between the measure of a drug concentration and the corresponding probability is given by a logistic equation of the form:

$$
P=\frac{e^{\log i t}}{1+e^{\log i t}}=\frac{1}{1+e^{-\log i t}}
$$

where $P$ is the binary or dichotomous outcome from 0 to 1, Logit $(L)$ is the natural log of the odds (relative risk):

$$
L=\operatorname{logit}(P)=\log (o d d s)=\operatorname{Ln} \frac{P}{(1-P)}
$$

Tbe Logit can be witten as a linear equation of the form:

$$
\text { Logit }=\theta_{1}+\theta_{2}(\text { predictor } 1)+\theta_{3}(\text { predictor } 2) \ldots
$$

This equation allows the development of linear models describing the relationship between the success probability and various predictors. The logit bearing linearity in its parameters $\left(\theta_{i}\right)$ and ranging from $-\infty$ to $+\infty$, it transforms the $0-1$ probability to a 
continuous scale. For example, the probability of cure for an antibiotic of the quinolone family can be modeled as

$$
\mathrm{POC}=\frac{1}{1+e^{-\left(\theta_{1}+\theta_{2} X\right)}}
$$

where POC is the probability of cure (from 0 to 1 ), $\theta_{1}$ is a parameter for baseline (here a placebo effect) and $\theta_{2}$ is an index of sensitivity (a slope). $X$ is the independent variable and can be the AUC/MIC ratio that is classically selected for this class of antibiotic to predict efficacy or any other quantitative index predicting drug efficacy. If $\theta_{2}$ equal 0 , the response is independent of $X$ and the POC corresponds to the placebo effect in Equation 13.14. Exponential $\theta_{2}$ represents the change in the odds of the outcome (multiplicatively) by increasing $X$ by 1 unit holding all other predictors constant. In this relationship, the equivalent of the $E C_{50}$ (or $E D_{50}$ ) of Equation 13.3 is named $E L_{50}$; it is now the median effective level of the independent variable (e.g., dose, AUC, AUC/MIC) for which $50 \%$ of the subjects are above the threshold, and the slope of the curve now represents the dispersion (variance) of the threshold in a population. The $E L_{50}$ can be estimated by the ratio $-\theta_{1} / \theta_{2}$, the $X$ value associated with the steepest slope of the $\mathrm{S}$-shaped logistic regression curve. When Equation 13.14 is plotted against a range of $X$ values, this model has an S-shaped curve, approaching values of 0 and 1 gradually as shown in Fig. 13.11.

This kind of model can be extended to include qualitative indicators (e.g., gender, breed, etc.). For the development of a binary logistic model see Fiedler-Kelly (2007). When the end point is measured on an ordered categorical scale with several levels such as pain scores or adverse events (mild, moderate, severe, or life threatening) with repeated measurement and censored data, more advanced models are in order as described by Ette et al. (2007).

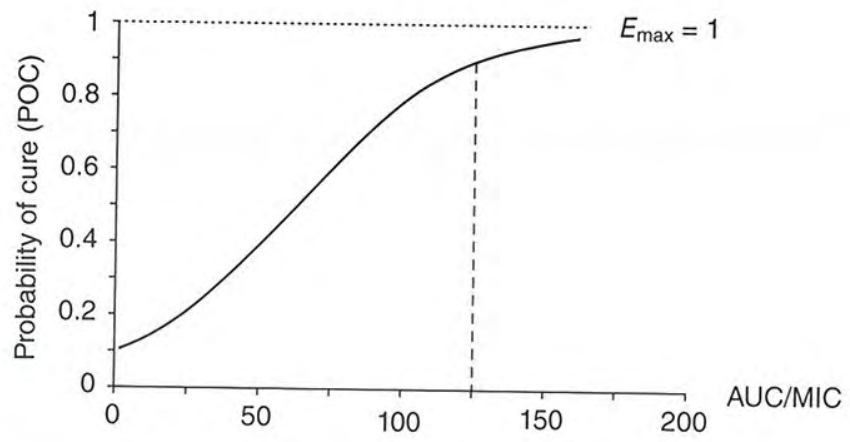

Fig. 13.11 Logistic regression. When an effect is binary (cure/no cure), a logistic curve is used to describe the relationship between the independent variable (often dose, exposure, or any explicative variable) and the dependent variable (a probability between 0 and 1). This quantal dose-response curve does not relate dose to intensity of effect but to the frequency in a population of individuals in which a drug produces an all-or-nothing effect. Here, the POC for a hypothetical concentration-dependent antibiotic has been plotted against the value of a PK/PD index generally selected for this class of antibiotics (i.e., AUC/MIC). The threshold AUC/MIC varies among individuals and the fixed effect model quantifies the likelihood or probability that a given AUC/MIC will produce a cure or not. 


\subsection{THE MEANING AND CLINICAL RELEVANCE OF THE DIFFERENT DEPENDENT VARIABLES OF A PD MODEL: ACTION, EFFECT, RESPONSE, BIOMARKERS, AND SURROGATES}

When performing a $\mathrm{PK} / \mathrm{PD}$ analysis, special attention should be paid to the nature of the dependent variable that has been selected. The dependent variable $E$ is named after the generic term "effect." However, it can be useful to distinguish between drug action, drug effect, and drug response. For example, for an antibiotic, the "action" consists of inhibiting bactcrial protein synthesis, the "cffect" corresponds to the killing rate of bacteria, and the clinical "response" would be the cure of animals. When the objective of a PK/PD trial is to investigate a mechanism of action, the drug action at a primary site (bacteria, parasites, enzyme, receptor) should be measured. However, if the purpose of a PK/PD trial is to determine a dosage regimen, it is more relevant to measure the response of clinical interest. For example, to explore the pharmacological effect and selectivity of NSAIDs, the convenient dependent variables are the level of inhibition of the different COX isoenzymes. However, if the goal of the PK/PD is to establish a dosage regimen for future clinical trials, then the reduction of an experimental lameness will be more relevant.

In many situations, it is impossible or too difficult to select the most meaningfu] end point as a dependent variable: for example, how dogs feels under an analgesic treatment may be difficult to assess quantitatively using demeanor and the effect of an anticancer drug on the survival time of treated dogs or cats may be too delayed to be conveniently used in a PK/PD nodeling strategy. For these situations, the end points of ultimate interest are replaced by so-called biomarkers that are assessed more easily and rapidly than definitive and more easily quantifiable clinical end points.

The role of biomarkers in PK/PD modeling is pivotal and needs to be fully understood. A biomarker, as defined by the Biomarkers Definition Working Group (Atkinson et al., 2001 ), is a characteristic that can be objectively measured and evaluated as an indicator of normal biological processes, pathogenic processes, or pharmacological responses to a therapeutic intervention as depicted in Fig. 13.12. Biomarkers are usually more precisely measured with validated assays or medical devices compared with clinical outcomes, thus making biomarkers attractive in preclinical PK/PD modeling. For example, biomarkers are extensively used in preclinical PK/PD modeling to assess NSAIDs' potency and selectivity by measuring the inhibition of $\mathrm{PGE}_{2}$ formation using an appropriate in vimo or ex vivo test system (Lees et al., 2004). Angiotensin converting enzyme (ACE) inhibition has been used to evaluate the potency of ACE inhibitors in dogs (Toutain et al., 2000) and cats (King et al., 2003). When the clinical end point of ultimate interest is difficult to quantify or is much delayed in time, it may be judicious to replace the clinical end point in a PK/PD trial by a surrogate end point, whicb is a biomarker expected to predict clinical benefit (or harm) based on epidemiological, therapeutic, pathophysiological, or other scientific evidence. The most commonly used surrogate end point for regulatory purposes is the plasma concentration of drug in bioequivalence studies. Some examples of surrogates in veterinary medicine are the PK/PD indices that have been proposed for predicting the clinical success and bacteriological cure of antibiotics such as AUC/MIC or $T>\mathrm{MIC}$, and conventional radiography for the evaluation of osteoarthritis. 


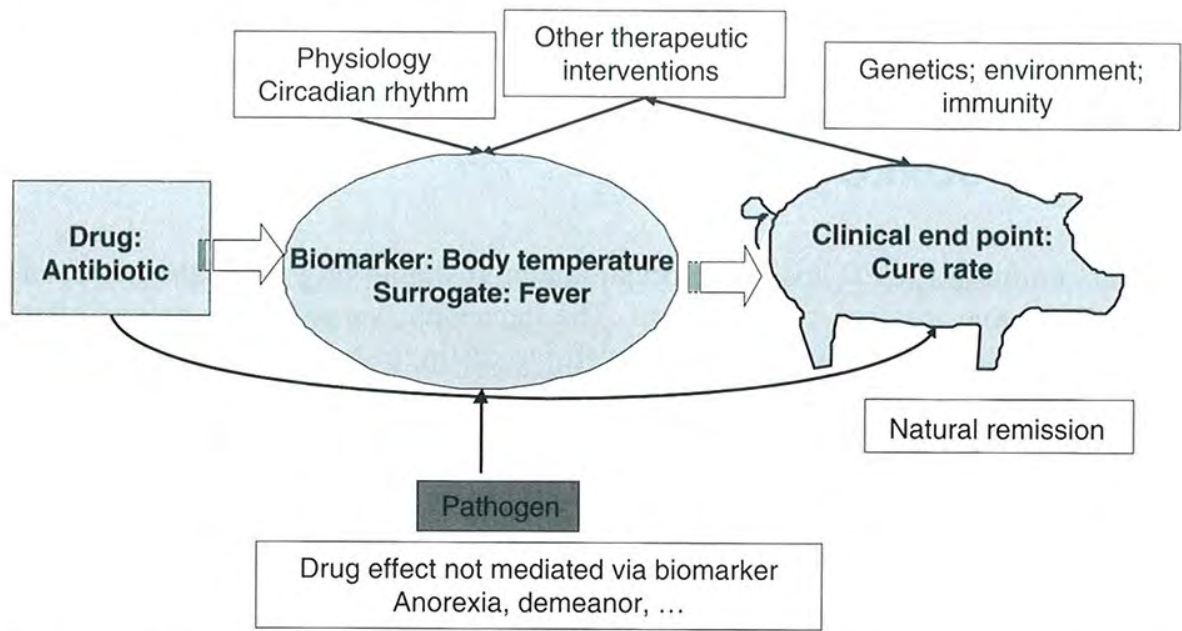

Fig. 13.12 Biomarker, surrogate, and clinical end points for an antibiotic intended to treat pneumonia in pigs. Hyperthermia should be considered as a biomarker, fever (i.e., hyperthermia, anorexia, drowsiness) should be considered as a surrogate, and the clinical cure rate should be considered as the clinical end point of ultimate interest.

Whatever the selected biomarkers, they should be carefully validated even if they appear to be directly linked to the disease of interest. Currently, few biomarkers in veterinary medicine are robust enough to serve as surrogate end points as is the case in human medicine for blood pressure and cholesterol concentrations that are accepted as surrogates for cardiovascular drugs. Indeed, a drug may have a favorable effect on a biomarker and an unfavorable effect on the disease and any biomarker believed to be a surrogate of clinical relevance must be validated. For a classification and validation of the different types of biomarker, see Williams and Ette (2007). In the future, it is likely that proteomics and metabolomics will provide some biomarkers of drug effects.

\subsubsection{Hill equation parameters}

Drug potency, maximal efficacy, and sensitivity are the three PD parameters that can be estimated using the Hill equation (Eq. 13.3). They have been defined in Figs. 13.6 and 13.7.

\subsubsection{Potency $\left(E C_{50}\right)$}

Potency expresses the intensity of drug activity in terms of concentration. $E C_{50}$ (for stimulation) and $I C_{50}$ (for inhibition) are estimated directly by the Hill model. Potency varies inversely with the concentration required to produce the effect (Fig. 13.6b). It should be stressed that a drug can be more potent but less efficacious than another one as is the case of buprenorphine versus morphine. However, provided that the required dose can be conveniently administered, potency alone is relatively unimportant from a therapeutic perspective. Low potency becomes a disadvantage only when the size of the effective dose renders it difficult to administer (e.g., for spot-on drugs in pets). 


\subsubsection{Maximal efficacy $\left(E_{\max }\right)$}

$E_{\text {nax }}$ is the maximum effect that can be generated by a particular system (e.g., the maximal possible reduction of blood pressure). It is the most important parameter for clinicians when the dependent measured variable has a clinical meaning (blood pressure, lameness score), but in most indirect effect models, $E_{\text {max }}$ does not have a direct meaning; for example, $E_{\text {max }}$ can be the scalar by which the modeled underlying process can be multiplied.

\subsubsection{Sensitivity}

In the Hill model, the shape coefficient $(n)$ gives the slope of the concentration-effect relationship (Fig. 13.5). In receptor theory, $n$ has a precise meaning in terms of drug binding, but in vivo, $n$ should not be interpreted in mechanistic terms. $n$ is of considerable clinical relevance when examining selectivity and drug sensitivity, that is, the range of useful concentrations (doses) for achieving a desired effect or avoiding an unwanted effect. Fig. 13.13 provides a more detailed explanation.

In vivo, for drugs with a low $n(<1)$, the PD profije is shallow, meaning that only moderate changes in effect will be observed over a wide range of drug concentrations (several orders of magnitude). This type of relationship explains the very long-lasting action of some drugs (e.g., beta blockers for heart rate). For this kind of drug, the length of the terminal half-life can be very important for predicting the duration of an effect. In contrast, for drugs with a steep slope, minor variations in the concentrations around $E C_{50}$ can produce

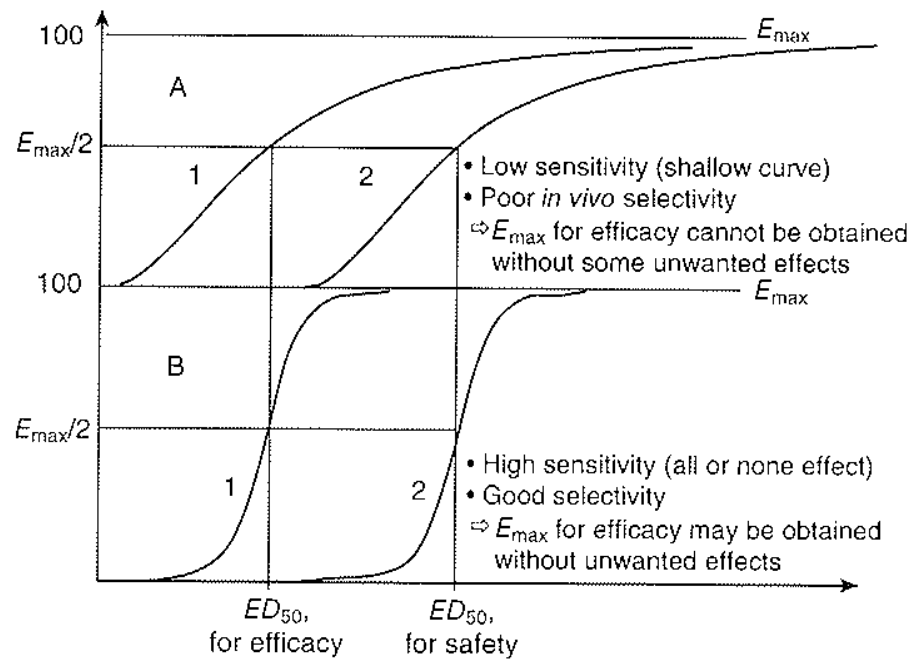

Fig. 13.13 In vivo drug selectivity is related to both the therapeutic index (i.e., the ratio $E D_{50}$ safety/ED 50 efficacy) ond to the slope of the concentration effect relationship. Drugs A (top) ond B (bothom) have the same potency $\left(E D_{50}\right)$ and the same efficacy (same $E_{\text {trax }}$ ) for the desired effect (curve 1) and unwanted effects (curve 2), thot is, the same theropeutic index. However, they differ in terms of sensitivity (slope) (shallow for drug $A$ and steep for drug B). Only with diug B can the full effect (i.e., $E_{\text {max }}$ ) be obtained without any significant side effects despite the fact that drugs $A$ and $B$ have the same $E D_{50}$ safety/ED 50 efficacy ratio. Definition of therapeutic index by $E D_{10}$ safefy/ED $D_{90}$ efficacy takes into account the differences in sensitivity of dose-response curves. 
effects ranging from null to nearly maximal. This is the case with phenylbutazone, flunixin, and meloxicam for lameness in horses. Drugs with a high $n$ but a low therapeutic index may require therapeutic drug monitoring to guarantee efficacy without toxicity. As $n$ increases $(n>5)$, the concentration range diminishes to become a simple threshold (i.e., critical concentration just above $E C_{50}$ ), and the graded PD model becomes a quantal model, representing a limit of graded concentration when $n \gg 1$.

\subsubsection{Modeling the PD baseline}

As quoted above, $E_{0}$ in Equation 13.3 may or may not be a constant. If the drug effect is investigated over a short period of time or if physiological stability of the measured effect can be assumed, $E_{0}$ is treated as a simple parameter. More generally, the baseline is subjected to some variations and it should be modeled. Many models, empirical or mechanistically based, can be selected or developed to model baseline status; the simplest is the linear model:

$$
\operatorname{Baseline}(t)=E_{0}+\alpha t
$$

where $E_{0}$ is the initial baseline status, $\alpha$ is a slope parameter, and $t$ is the time after the initial observation.

If the baseline undergoes a circadian rhythm such as, cortisol secretion from the adrenal gland, modeling the suppressive effect of exogenous glucocorticoids should not be confounded with the occurrence of a natural nadir (physiological minimal plasma cortisol concentration) and the baseline cortisol plasma concentration can be modeled as follows:

$$
E_{0}=E_{m}+E_{b} \cdot \cos \left[\left(t-t_{z}\right) \cdot 2 \pi / 24\right]
$$

where $E_{0}$ characterizes the circadian pattern of plasma cortisol concentrations (actually a production rate), $E_{m}$ and $E_{b}$ (same units as $E_{0}$ ) are the cosine function mesor (i.e., the adjusted mean) and amplitude, $t$ is the clock time converted with the numeric fraction into radians $t_{z}$ is the peak time (acrophase), and $2 \pi / 24$ is used to convert time into radians. Then a full model for the synthetic glucocorticoid suppressive effect can be developed by mitigating $E_{0}$ with an $I_{\max }$ model.

Currently, most PD models are mechanistically founded and the baseline (response in absence of drug) cannot be summarized by a single $E_{0}$ parameter but is the result of a dynamic equilibrium that can be affected not only by drug action but also by other factors including experimental challenge or spontaneous (disease) progression. This aspect will be developed later in the chapter.

\subsubsection{The PK models}

In a PK/PD modeling approach, a PK model is required to serve the role of the independent (or explicative) variable. The PK model (plasma, urine, or any other relevant matrix) can be a classical one (e.g., a compartmental model as developed in Chapter 8) for which parameters have been previously estimated. Its role is only to provide concentration inputs 
in the PD model. Thus, it can he replaced by a noncompartmental model presented in Chapter 9 or simply a curve-smoothing procedure (e.g., cubic spline that is a special type of piecewise polynomial) if the plasma concentration profile cannot he described adequately by a conventional PK model. This is a nonparametric approach that can be extended to the PD part of the model. In PK/PD modeling, the PK step is generally the easiest to carry out, but a more advanced PK model may be required if the predicted effect is related not only to the parent drug but also to some active metabolite(s), or if a racemate drug is administered with an enantioselective disposition. For example, to build a PK/PD model of the analgesic effect of ketoprofen, it is important to consider that both the disposition and the potency of the $R(+)$ and $S(-)$ enantiomers are different, so that both enantiomers must be explicitly included in the PK/PD model rather than just the total ketoprofen plasma concentration. Conversely, when no concentration data are collected and when the only information is the kinetics of the response, a so-called kinetic-pharmacodynamic model (K-PD) can be built. In this case, the plasma concentrations are unknown but the input rate function is modeled using a simple monocompartmental model and the dosing history (for details see Pillai et al., 2004).

\subsection{HYSTERESIS}

\subsubsection{The origin of the delay in drug action for the building of a PK/PD model}

For some drugs, the plasma concentration profle as generated by the PK model and the observed effects can be in phase, and the plasma concentrations can be directly incorporated into a PD model as described in Equation 13.3. Levy (1964), a pioneer of PK/PD modeling, used a monocompartmental model (first-order kinetics) to describe d-tubocurarine disposition. When introducing the plasma concentrations into a simple log-linear model (Eq. 13.7), he was able to describe correctly the time course of muscle paralysis. It can be easily shown that the effect versus time is a simple linear function of time (zero-order kinetic). Such quite simple PK/PD models were historically developed especially for cardiovascular drugs (effect of digoxin on systolic time intervals, effect of disopyramide and prolongation of the Q-T interval).

Most often plasma concentration profiles and the observed effects are not in phase, and the plasma concentrations cannot be directly incorporated into a PD model. For most drugs, the effect lags behind the plasma concentrations. This can easily be visualized by plotting the effects ( $y$-axis) against the plasma concentrations ( $x$-axis). When data points follow a chronological order; a loop is observed as seen in Fig. 13.14. This phenomenon is termed "hysteresis," from the Greek word meaning "coming late." The inverse situation (i.e., a lesser effect at a later time for the same plasma concentration) is termed "proteresis," a neologism meaning "coming early." This terminology is preferred to "clockwise hysteresis" and "counterclockwise hysteresis," which is often used in the literature. The actual direction of the loop depends on the direction of the effect (positive or negative).

When a hysteresis loop is observed, the cause of the delay must be identified to select a modeling strategy. Observing the time of occurrence of the maximal effect when the dose of the tested drug is progressively increased, as depicted in Fig. 13.15, may also help to select a model. 

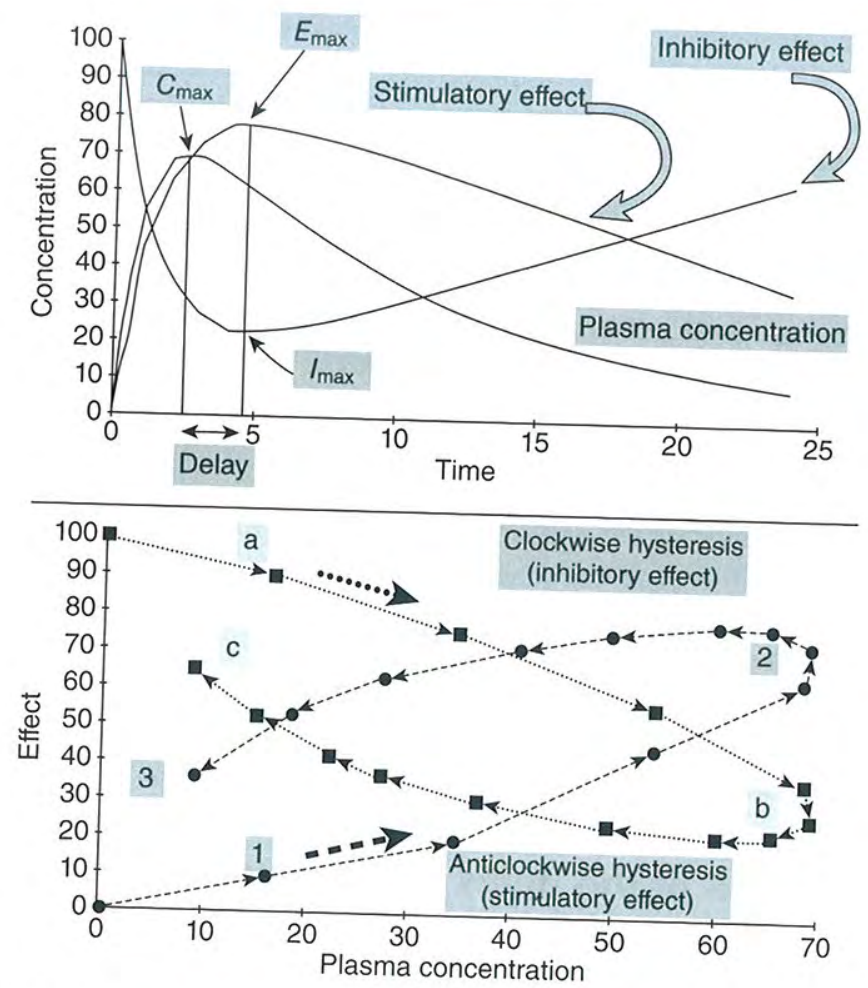

Fig. 13.14 Lack of synchronization of concentration-effect relationship and hysteresis plot. In general, plasma concentration-time and effect-time relationships are not in phase. The peak plasma concentration $\left(C_{\max }\right)$ occurs before a peak effect $\left(E_{\max }\right.$ for a stimulatory effect and $I_{\max }$ for an inhibitory effect). This sible. Hysteresis is well evidenced by plotting given concentration, two different levels of effect are posthis example, the plot reveals two hysteresis lasma concentration versus effect in a time sequence. In (from 1 to 3) and a clockwise effect (from a to c) for the inhibitory effect.

When the delay is of PK origin (e.g., slow rate of distribution at the biophase, transformation of a prodrug into its active metabolite) and when the drug effect is directly related to the drug concentration at the biophase level, an effect-compartment model can be chosen with the selection of a link model. However, for most drugs, the measured response is not a primary drug action resulting from direct binding of the drug to its receptor. Rather, there is a cascade of time-consuming biological events between the plasma drug concentration and the final observed response (e.g., signal transduction and other processes that are discussed below). Under these conditions, the observed delay between the kinetics of the plasma concentrations and the time development of a response is not of distributional origin (i.e., of PK origin) but rather reflects the intrinsic temporal responsiveness of the system that is of PD origin. For this kind of response, so-called indirect response models are suitable. In the next section, we will see how to build a PK/PD model when the delay is either

Fig. 13.16 gives the general decision tree to select a class of model according to the origin of the delay between the plasma concentration versus time and the effect. 

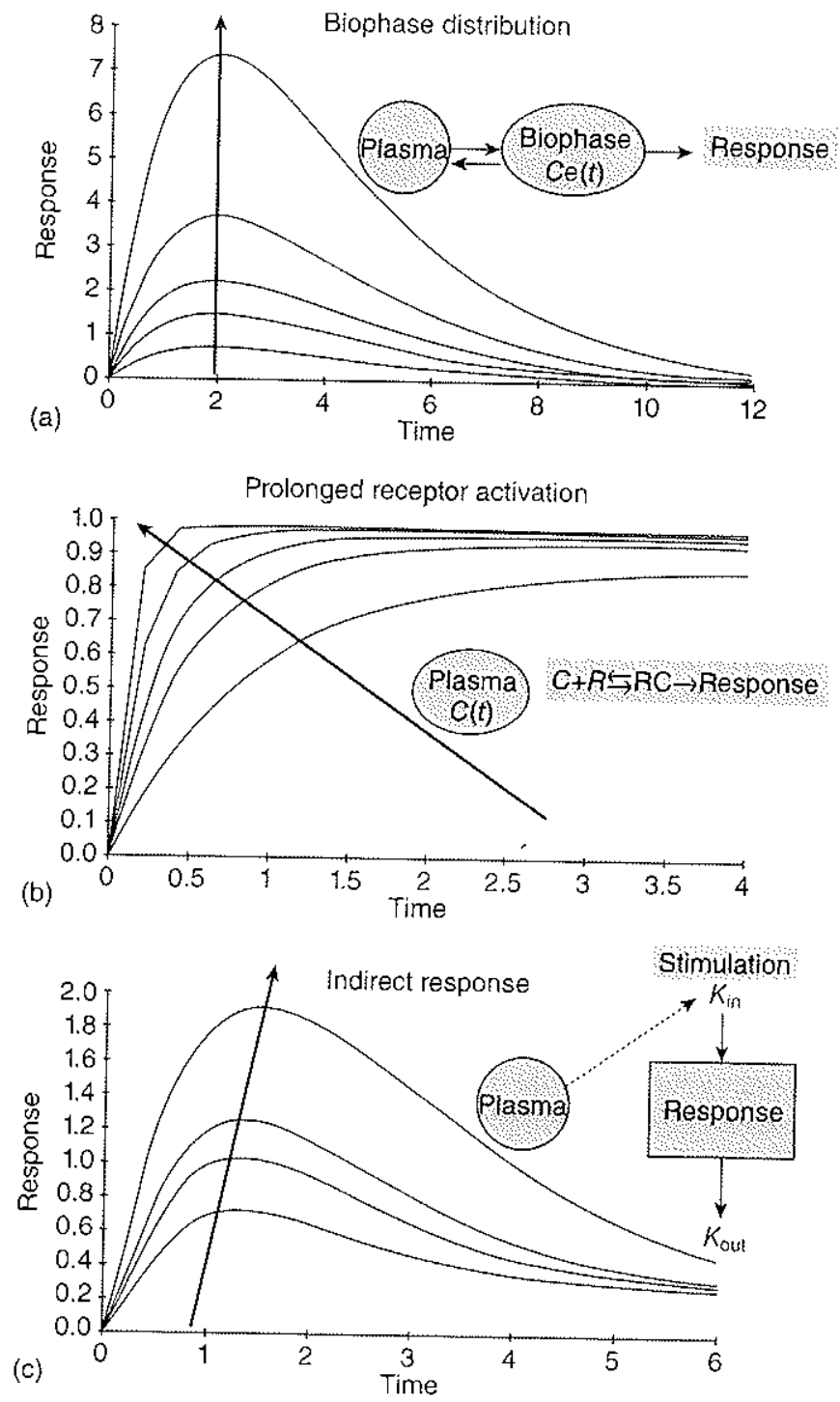

Fig. 13.15 Influence of the dose level on the time of maximal effect according to the fype of the selected model. (a) For an effect compartment model (distributional model), the time of maximal effect is independent of the dose level. (b) For a prolonged receptor activation model (RC is the drug-receptor complex and $R$ the free receptars), the time of moximum effect is observed earlier and earlier when the dose is increosed. (c) The inverse phenomenon is observed with an indirect effect model, that is, a delay when the dase is increased.

\subsubsection{Delay of PK origin and the link model}

If it is acknowledged that the Imiting step for a drug to produce its effect is of PK origin with a rather slow distributional process followed by an immediate action when the drug has gained access to its biophase, the biophase can be represented by a specific compartment named the "hypothetical effect compartment" as historically introduced by Sheiner 


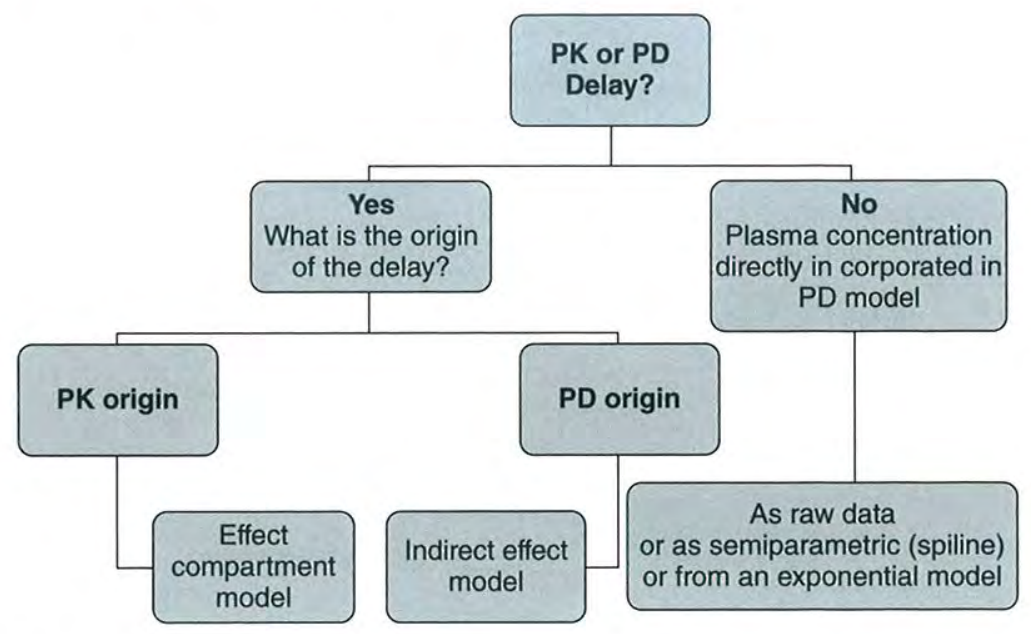

Fig. 13.16 Decision tree to select a PK/PD model according to the origin of the delay between the plasma concentration and observed effect.

et al. (1979) and shown in Fig. 13.17 describing the time development action of d-tubocurarine. Here, the hypothetical compartment was introduced as a tool to model the delayed effect by using the time course of the effect itself to define the rate of drug movement into the biophase. To accomplish this, the effect compartment should have two main features: (1) there is no hysteresis between the drug concentration in the effect compartment and the effect, meaning that the drug effect is instantaneously reversible and is a memoryless function of biophase drug concentration and (2) the amount of drug entering the compartment is negligible and does not affect the ability of the PK model to describe the time course of systemic drug concentrations. Since the amount of drug entering the effect compartment is assumed to be negligible, any drug entering the effect compartment can be eliminated directly from that compartment (according to a first-order rate constant named $K_{\mathrm{e} 0}$ ), rather than returning to the central compartment for systemic elimination. This assumption greatly simplifies the mathematical expression of the model that allows $E_{\max }$, the plasma steady-state $E C_{50}$ (i.e., PD parameters), and $K_{\mathrm{e} 0}$ (the link model parameter) to be estimated from PD versus time data using nonlinear regression techniques. For a detailed description of this model, see the first edition of this book and the review by Holford and Sheiner (1982). Such a model was used to describe the effect of meperidine in goats (Qiao and Fung, 1993). Today, it is acknowledged that most delays are not of PK but are of PD origin, and this kind of model is used much less often.

\subsubsection{Modeling delay of PD origin using physiological systems}

It is now acknowledged that the limiting step for a drug to produce its effect is most often of a PD origin with a rather rapid distributional process followed by a slow development of the observed drug response when the drug had gained access to its biophase. This is due to the drug mechanisms of action at the cellular level and/or the drug effect on the physiological or pathophysiological system. For example, suppression of lameness by an NSAID requires inhibition of a COX isoenzyme at the biophase level (e.g., joint articulation) and 


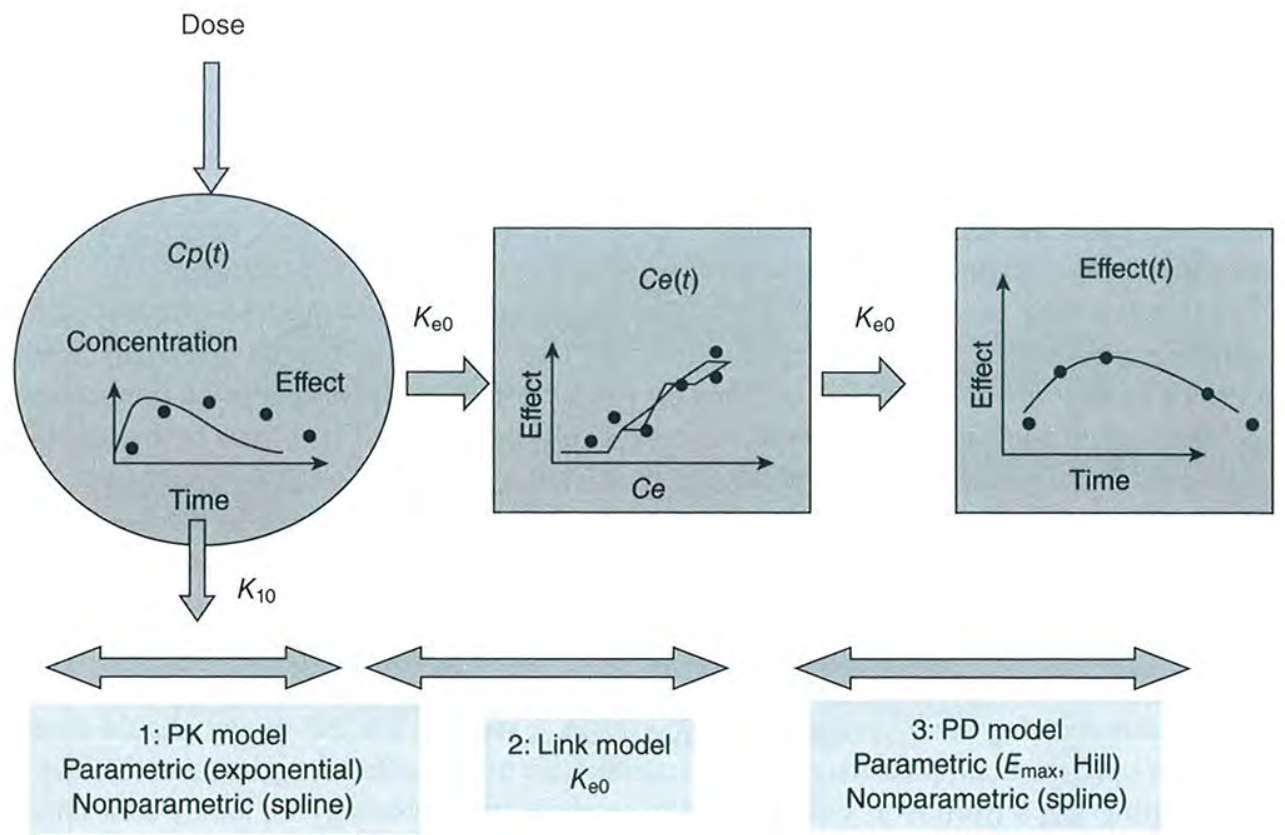

Fig. 13.17 The "effect compartment model" was proposed to model situations where the delay between plasma concentration, $C(t)$, and observed pharmacological effect, $E(t)$, are due to a distributional delay from time taken for the drug to gain access to its biophase. The model removes this delay by creating a hypothetical effect compartment in which the drug concentration $\mathrm{Ce}(t)$ is in phase with $E(t)$. Schematically, this model is formed by three components: a classical compartmental PK model, a "hypothetical effect compartment," and a PD model. $C(t)$ and $\mathrm{Ce}(t)$ are drug concentrations in the central and hypothetical effect compartments, respectively. The drug is administered and eliminated from the central compartment with $K_{10}$ as the rate constant of elimination; the drug gains access to the effect compartment through a first-order rate constant historically called $K_{1 \mathrm{e}}$ (but now equated to $K_{\mathrm{e} 0}$; see later). This "virtual effect compartment" should only receive a negligible amount of drug in order to have no influence on the overall drug disposition as described by the main compartmental model. This condition eliminates the drug directly out of the effect compartment (biophase) through a first-order rate constant that renders simpler equations describing $\mathrm{Ce}(t)$. This exit rate constant is named $\mathrm{K}_{\mathrm{e} 0}$ and it controls the rate of drug equilibration in the effect compartment. $K_{\mathrm{e} o}$ should be selected (estimated) in order to have no hysteresis between $\mathrm{Ce}(t)$ and the pharmacological effect. $\mathrm{Ce}(t)$ cannot be directly measured, but rather is indirectly derived from the time course of the drug effect itself that by essence, is parallel to $\mathrm{Ce}(t) . \mathrm{K}_{\mathrm{e} 0}$ is the link model, $K_{\mathrm{e} 0}$ combined with $C(t)$ - the plasma kinetics-enables $\mathrm{Ce}(t)$ to be computed. In this model, $K_{\text {le }}$ was equated to $K_{\mathrm{e} o}$ not because $C(t)$ is equal to $\mathrm{Ce}(t)$ in steady-state conditions but for identifiability reasons. The consequence is that the $E C_{50}$ (or the $I C_{50}$ ) that is computed in the last step of the modeling is actually the steady-state plasma concentration corresponding to half the maximum effect and not the actual $E C_{50}$ (or $I C_{50}$ ) at the biophase level. To operate, the effect compartment model should be in rapid equilibrium with $C(t)$. However, if $K_{\mathrm{e} 0}$ is slow (compared with the other rate constant of the model), the biophase is not in equilibrium with $C(t)$ and the $C e(t)$ profile would not be reflected by $C(t)$. The last part of the model is the PD model ( $E_{\max }$, Hill model). To use this model, one needs PD data from the rise and fall of the pharmacological effect over time to characterize correctly the time development of $\mathrm{Ce}(t)$. From this modeling, the half-time of equilibration can be computed from $K_{\mathrm{e} 0}$ (i.e., $0.693 / K_{\mathrm{e} 0}$ ). A feature of this model is that the $T_{\max }$ of the observed effect is dose independent, whereas it is time dependent for the indirect effect model (see Fig. 13.15). 
then, the time-consuming elimination of the proinflammatory substances present before the drug administration. For many drugs, the effect involves some protein synthesis and a cascade of different events reflecting the complexity of the targeted system. This is the so-called pharmacological transductions that govern the transduction of target activation into a final drug response in vivo.

When the drug concentration is directly incorporated into a PD model without specific consideration for the system under investigation, the model is said to be empirical: these types of models are a model of data. They may be able to describe properly the time course of effect when little is known about the underlying process and they can be reasonably predictive if they are used in conditions rather similar to those already studied (e.g., for interpolation). When a PK/PD model includes a submodel that is related to the biology of the system itself, independently of the drug under investigation and with its own structural parameters, the PK/PD model is said to be semimechanistic or mechanistic, depending on its level of complexity. Here, the objective is not only to describe empirically what is the output (effect) from a black box (body) for a given input (dose), but also to understand the in vivo pharmacology and systems biology. Hence, a suitable PK/PD model should determine not only the main pharmacodynamic parameters of the tested drug but also the major rate-limiting steps (turnover, transduction, tolerance) in the biology of the system under investigation. Fig. 13.18 depicts the current view of the different basic components that might be included in a PK/PD model.

Mechanistic models are more complex and more fully described by the different components of a system, as is the case of a physiologically based pharmacokinetic model (PBPK) as discussed in Chapter 11. For antibiotics, anticancer drugs, and parasiticides, the drug effect consists of some irreversible inactivation and to do so the natural proliferation of the target in the absence of drug should be modeled.

The most used physiological PD model is the so-called turnover model. This model is based on the established fact that most physiological and biochemical functions are in dynamical equilibrium with two terms: a term corresponding to the formation of the response and a term corresponding to the loss of the response. The rate of change of response over time with no drug can be expressed as follows:

$$
d R / d t=K_{\text {in }}-K_{\text {out }} \cdot R
$$

where $d R / d t$ represents the rate of variation in the response variable $(R) . K_{\text {in }}$ is the rate of input, and $K_{\text {out }} \cdot R$ is the rate of loss; the model assumes that the measured response is being formed at a zero-order constant rate $\left(K_{\text {in }}\right)$ but disappears in a first-order manner $\left(K_{\text {out }}\right)$.

In control conditions (no drug, no pathological challenge), $d R / d t=K_{\text {in }}-K_{\text {out }} R=0$, for example, there is no change in the response variable and thus $K_{\text {in }}=K_{\text {out }} R_{0} . K_{\text {in }}$ can reflect the production rate of a measurable endogenous substance (hormone, mediator) with a dimension of concentration per unit of time and $K_{\text {out }}$ is a hybrid rate time constant having a dimension of $1 /$ time with the same meaning as $K_{10}$ for a monocompartmental model (see Chapter 8) that is proportional to the clearance of that substance and inversely proportional to its volume of distribution. $K_{\text {in }}$ and $K_{\text {out }}$ may or may not have a precise meaning: when modeling the antipyretic effect of NSAIDs using body temperature as a surrogate, $K_{\mathrm{in}}\left({ }^{\circ} \mathrm{C}\right.$ per time units) indirectly reflects thermogenesis (watts) and $K_{\text {out }}$ (per time units) indirectly reflects thermolysis (watts) even if for a physiologist, thermolysis cannot be reduced to a 
1: Dose titration

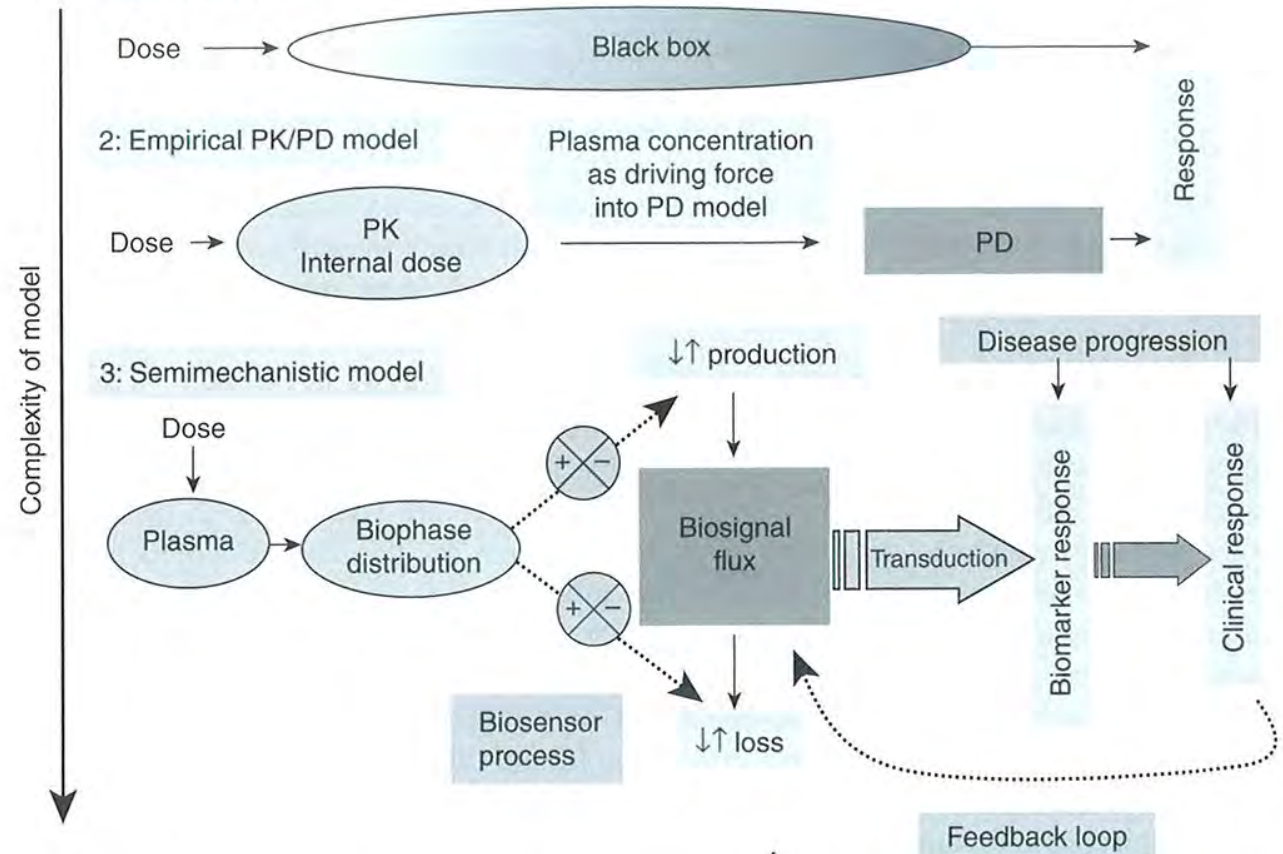

Fig. 13.18 Different levels of complexity of modeling the dose-effect relationship. The simplest model is the dose-effect relationship: it is typically a black box approach with the dose as the explicative variable and the clinical response as the dependent variable linked by a simple model (statistical or PD). The events between the dose and the response (e.g., PK and PD processes) are totally ignored. The black box can be opened to account for the two main steps separating a dose from its effect, namely a PK and a PD step. The main difference between a dose-titration model and an empirical PK/PD model is that now the plasma concentration profile is the explicative variable (the driving force) explaining effect and response. Both the PK and PD steps can be broken down further to characterize the relationship between a dose and its response in a more mechanistic way. For example, the PK step can include a distributional phase by including an effect compartment (biophase) with a link model; it is the PD step that is the most explicitly modeled. The drug acts on some biosensor process involving the reversible or irreversible interaction between the drug and its pharmacological target (receptor binding, killing pathogens) and may be described or not by various receptor occupancy models or some irreversible model of drug target interaction. Many drugs act via some indirect mechanisms on system-related processes and the biosensor may influence the production or loss of endogenous mediators (biosignal flux). This altered flux of mediators may not represent the observed terminal effect but rather trigger a further pharmacological transduction process (e.g., second messenger cascade), thus accounting for a further time delay. In addition, the system itself on which the drug is acting may be time variant, requiring, for example, the disease progression to be modeled or is able to trigger some feedback loop requiring additional modeling components.

passive phenomenon described by a first-order process. For this end point, the action of an NSAID will be to increase $K_{\text {out }}$ rather than decrease $K_{\text {in }}$ because it has been shown that NSAIDs stimulate thermolysis mechanisms. However, if the anti-inflammatory effect of the same NSAID is described using a quantitative lameness scoring as a biomarker, $K_{\text {in }}$ will reflect more loosely all the different underlying mechanisms, triggering pain associated 
with inflammation (local release of inflammation mediators, translation into pain including detection and modulation by the central nervous system of the pain signal). $K_{\text {out }}$ will reflect every mechanism mitigating lameness (such as elimination of the inflammatory mediators, modulation of pain) and it can be more difficult in this case to decide if the NSAID is acting mainly by decreasing $K_{\text {in }}$ and/or also by increasing $K_{\text {out }}$.

\subsection{TURNOVER MODELS}

In an empirical PK/PD model, the status of the biological system in the absence of drug is kept invariable with time, but this is not realistic when a drug is acting on a disease that deteriorates over time or when an animal is subjected to an experimental challenge as an experimental inflammation or infection. Turnover models are well suited to incorporate other submodel elements accounting for a placebo effect, for the time development of an experimental challenge (such as experimental inflammation) or to take into account the natural progression of a physiological process (e.g., growth) or pathological condition (e.g., diabetes, cancer). Several modeling strategies can be selected according to the type of drug action, namely purely symptomatic (no effect on the disease itself), disease-modifying effect (protective effect), or curative effect. See Mould (2007) and Post et al. (2005) for further discussions.

For a placebo effect the following equation can be used:

$$
d R / d t=K_{\text {in }}+\text { Placebo }(t)-K_{\text {out }} \cdot R
$$

where placebo can be modeled as:

$$
\text { Placebo }(t)=\mathrm{P} 1\left[e^{-\mathrm{P} 2(\text { time-lag })}-e^{-\mathrm{P} 3(\text { time-lag })}\right]
$$

where P1, P2, and P3 are parameters of the input rate function allowing, after a given lag time (lag), introduction of a placebo-associated input rate that is first increasing and then decreasing if P1 is positive, or conversely, a placebo effect that is first decreasing and then increasing if P1 is negative. An example is the anticipation and habituation behavior that are observed when sequentially measuring pain responses when experimentally testing an analgesic drug in dogs.

In preclinical investigations, it is usual to use some experimental models to test new drugs; for example, experimental paw inflammation can be induced by administering a phlogogenic agent such as kaolin to assess the anti-inflammatory and antipyretic action of NSAIDs (Giraudel et al., 2005). This experimental inflammation is not steady and its time course should be typically modeled with a function able to account for its onset and its spontaneous recovery. This is normally done during the control period of a twoperiod crossover design in which the same animal is challenged with and without the tested drug.

For example, the time development of the inflammation or any pathophysiological response in a control period as assessed by a clinical end point such as hyperthermia, or lameness following a kaolin administration, can be modeled using an empirical biexponential equation of the same form as for a placebo: 


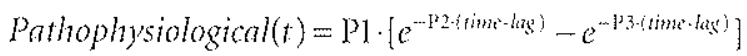

with P1, the intercept, as a scale factor reflecting the amplitude of the inflammatory response (as measured by hyperthermia, lameness, pain); P2 (1/time unit) is the slope of the decreasing phase of kaolin phlogogenic effect; P3 (1/time unit) is the rate constant refecting the increasing phase of the phlogogenic effect following the kaolin administration, and lag is the time of kaolin administration. The time development of the end point in the absence of drug during the control period is then modeled with:

$$
d R / d t=K_{\text {in }}+\text { Placebo }(t)+\text { Pathophysiological }(t)-K_{\text {out }} \cdot R
$$

In this additive equation, Placebo(t) and Pathophysiological( $t)$ are the input rate functions and have the same dimension as $K_{\mathrm{m}}$.

Finally, the drug effect can be introduced using a function mitigating pathophysiological( $t)$ as:

$$
d R / d t=K_{\mathrm{in}}+\text { Placebo }(t)+\text { Pathophysiological }(t) \cdot(1-\text { Drug })-K_{\mathrm{ou}} \cdot R
$$

where Drug is the PD model of drug action mitigating the inflammatory process.

To be identifiable, that is, to be in position to uniquely estimate every parameter of the model, the response has to be measured: (1) in control conditions to obtain information on $K_{\text {in }}$ and $K_{\text {ous }} ;(2)$ in a test placebo condition 10 get specific information on Placebo(t); (3) in a test condition but without drug to get genuine information on Pathophysiological(t); and finally, (4) in a test drug condition. This requires a relatively demanding crossover design. When all the data are collected under these different conditions, they are fited altogether assuming that the time course of the placebo effect is the same with and without inflammation and in the presence or not of the tested drug. This assumes a good reproducibility of the end points that are measured during different periods of a crossover design or reproducibility between groups if using a parallel design.

Other additive parameterization models are possible. To test the antipyretic effect of a drug using an experimental fever triggered by injection of an endotoxin, assuming that the endotoxin stimulates themogenesis $\left(K_{\mathrm{in}}\right)$, a maltiplicative function can be introduced in the general tumover system and can be written as:

$$
d R / d t=K_{\mathrm{int}} \cdot(1+\text { Pathophysiological }(t))+\text { Placebo }-K_{\text {out }} \cdot R
$$

For a PK/PD model developed in clinical rather than an experimental setting, the same class of equations can be used, with a model of disease progression replacing the pathophysiological function as:

$$
d R / d t=K_{\text {in }}+\text { Placebo }(t)+\text { Disease }(t)-K_{\text {out }} \cdot R
$$

Many options exist to model disease progression where different types of models are proposed for symptomatic, discase modifying, and curative drug action. 


\subsection{INCORPORATION OF A PD MODEL OF DRUG ACTION INTO A PHYSIOLOGICAL MODEL}

The incorporation of a PD model in an existing physiological model portrays the last step of the PK/PD modeling efforts that is a PD model can be nested in the different parts of a physiological model. For the turnover model (see Eq. 13.17), it is generally assumed that indirect drug action, consisting of inhibiting or stimulating physiological factors, control production, or dissipation of the measured effect (Dayneka et al., 1993). Inhibition or stimulation of the response production (or dissipation) can be described by accounting for inhibitory or stimulatory processes:

$$
\begin{aligned}
d R / d t= & K_{\text {in }} \cdot\{\text { stimulation or inhibition function }\} \\
& -K_{\text {out }} \cdot\{\text { stimulation or inhibition function }\} \cdot R
\end{aligned}
$$

or more formally:

$$
d R / d t=K_{\text {in }} \cdot\left\{1+H_{1}(t)\right\}-K_{\text {out }} \cdot\left\{1+H_{2}(t)\right\} \cdot R
$$

where $H(t)$ is a function of time. An inhibitory process can be described by the function $I(t)$ :

$$
I(t)=-\frac{I_{\max } \cdot C(t)}{I C_{50}+C(t)}
$$

where $I C_{50}$ is the drug (plasma) concentration that produces $50 \%$ of the maximum inhibition; $I_{\max }$ is a number from 0 to 1 ( 1 for total inhibition); and $C(t)$ is the drug (plasma) concentration over time.

By incorporating this function into Equation 13.17, we get two basic inhibitory PD models, as expressed:

$$
\begin{aligned}
& d R / d t=K_{\text {in }}\left(1-\frac{I_{\max } \cdot C(t)}{I C_{50}+C(t)}\right)-K_{\text {out }} \cdot R \\
& d R / d t=K_{\text {in }}-K_{\text {out }}\left(1-\frac{I_{\max } \cdot C(t)}{I C_{50}+C(t)}\right) \cdot R
\end{aligned}
$$

The model shown in Equation 13.28 stands for an inhibition of the response production rate (e.g., the action of a synthetic glucocorticoid on the secretion rate of cortisol by the adrenal gland). The model shown in Equation 13.29 stands for an inhibition of the response loss rate (e.g., the inhibition of $\mathrm{Na}^{+}$reabsorption by furosemide in the loop of Henle).

A stimulation process can be described as:

$$
S(t)=\frac{S_{\max } \cdot C(t)}{S C_{50}+C(t)}
$$


where $S C_{50}$ is the drug plasma concentration producing $50 \%$ of the maximum stimulation that is of $S_{\text {max }}$; $S_{\text {nax }}$ is a positive number and $C(t)$ is the drug (plasma) concentration over time. It should be noted that $S_{\text {max }}$ does not represent a maximum observed effect but a maximum for the stimulation of a plysiological process.

Incorporating the stimulatory function in Equation 13.17 gives two basic stimulatory PD models:

$$
d R / d t=K_{\text {in }}\left(1+\frac{S_{\max } \cdot C(t)}{S C_{50}+C(t)}\right)-K_{w u 1} \cdot R
$$

and

$$
d R / d t=K_{\mathrm{in}}-K_{\mathrm{out}}\left(1+\frac{S_{\max } \cdot C(t)}{S C_{50}+C(t)}\right) \cdot R
$$

The model shown in Equation 13.31 corresponds to the stimulation of the response production rate (e.g., production of CAMP by a bronchodilatator beta 2-agonist). Equation 13.31 was used in Fig. 13.19 to model the LH response to GnRH, the LH response being selected as a surrogate end point to detemine a dose able to reestablish estrous cycles in cows with ovarian cysts. The model shown in Equation 13.32 corsesponds to the stimulation of response loss (e.g., the antipyretic effect of NSAIDs with stimulation of thermolysis). These models have been used successfully for different classes of drugs (anticoagulants, corticosteroids, beta-adrenergics, antipyretics, etc).

\subsection{TIME-VARIANT MODELS: TOLERANCE AND REBOUNDS}

For a repeated drug administration, the PK/PD model might be able to adequately describe data for the first-dose administration, but progressively suffer some drift, that is, time dependency (nonstationarity). This is due to a physiological adaptation of the biological system with initiation of more or less complicated feedback mechanisms following repeated exposure to a drug. For instance, when a drug is given repeatedly, there can be a progressive reduction in the response to the drug due to some desensitization at the receptor level (downregulation). This phenomenon is called "tolerance" and it corresponds to the reversible decrease of the response for a given fixed plasma concentration. "Rebound" is the opposite effect that is observed at the cessation of drug administration.

Tolerance is frequently reported in human medicine but few relevant examples exist in veterinary medicine. Tolerance to opioids has been well described in animals but these drugs are seldom used for a long-enough period to have clinical consequences. Several mechanisms can explain this dampening phenomenon and they can be of a $\mathrm{PK}$ and/or PD origin. For phenobarbital that is extensively used as an anticonvulsant in small animals, tolerance develops rapidly, owing to the induction of hepatic enzymes increasing the phenobarbital metabolism. In addition, during a more prolonged treatment, there is a desensitization (downregulation) of GABA receptors. The formation of antagonistic metabolites competing with a drug for the same binding sites on the receptor and biofeedback regulation (counterregulation) are other tolerance mechanisms. Both empirical and more mecha- 

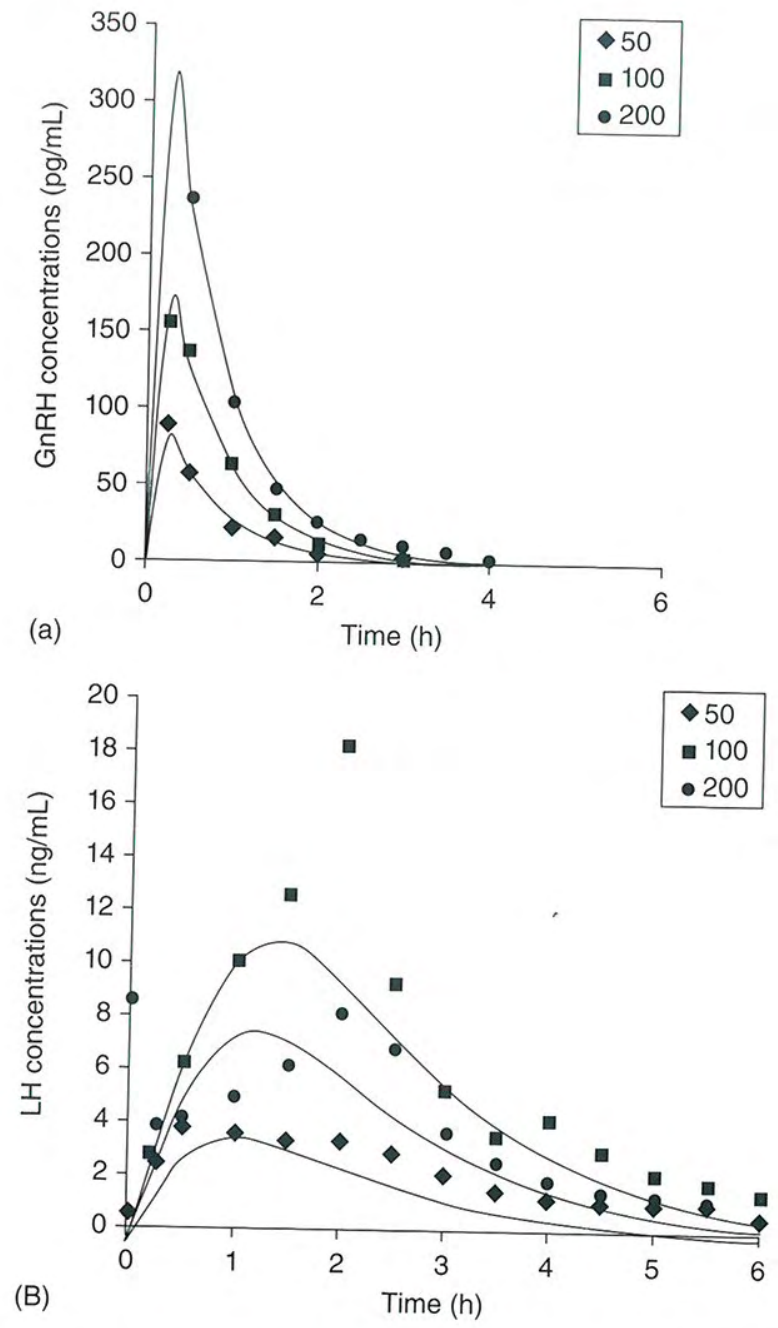

Fig. 13.19 PK/PD analysis of the luteinizing hormone (LH) response to gonadotropin-releasing hormone $(\mathrm{GnRH})$ in a cow. The pharmacokinetics of $\mathrm{GnRH}$ and the pharmacodynamic LH response were determined after administration of $\mathrm{GnRH}$ at doses of 50,100 , and $200 \mu \mathrm{g}$ in toto (see Fig. 13.4). The three doses were fitted together using an indirect effect PK/PD model. Observed and fitted values for $\mathrm{GnRH}$ (a) and plasma (LH) concentrations (b) for a representative cow. From this model, PD parameters were calculated, thus enabling a $\mathrm{GnRH}$ dose to be determined that reestablished estrous cycles in cows
with ovarian cysts.

nistically oriented models of tolerance have been developed. For example, $E_{\max }$ or $E C_{50}$ can be modeled to become time-dependent parameters, that is, to model a time dependency of efficacy (for a noncompetitive antagonistic inhibition) or a time dependency of drug potency (for a competitive antagonistic competition). A hypothetical tolerance counteracting compartment with its own constant can be introduced in the model to mimic the time course of tolerance. A compilation of approaches to modeling tolerance and rebound is given by Gabrielsson and Weiner (2006), though many of them are indistinguishable under different experimental conditions. 


\subsection{POPULATION PK/PD MODELING APPROACHES}

For any given species, the sources of PK variability in veterinary medicine (e.g., breed, age, sex, dietary factors, and kidney and liver functions) have been widely discussed, but the sources of PD variability have been little considered. It is now recognized that PD variability can be more pronounced than that associated with PK. This is especially true for antibiotics, where the clinical response is affected not only by the ability of the drug to reach the site of infection but also by the PD variability (host response to the invading pathogen and bacterial susceptibility). The reader should consult Chapter 16 for development of the statistical nomenclature used in this discussion.

One of the main advances of PK/PD approaches has been to separate the two main sources (PK and PD) of variability through the use of population PK/PD approaches. Similar to the PK population model, a population PK/PD model describing a drug effect across individuals and disease subgroups can be built. Using these techniques, population analysis can explain the variation between animals (or between groups of animals) not only in terms of drug exposure but also in terms of drug responsiveness. For example, the $E C_{50}$ of Equation 13.3 can be modeled using an exponential error model:

$$
E C_{50}=\theta_{2} \cdot e^{\eta_{2}}
$$

where $\eta$ is the deviation from the mean for the $i$ th subject (intersubject variability) with zero mean and variance $\omega^{2}$ enabling the interanimal variability of $E C_{50}$ in the population to be determined.

\subsubsection{Some practical considerations when building a PK/PD model}

The analysis of PK/PD data can be complex and time-consuming because it requires competent modelers having both a good understanding of the general principles of modeling (i.e., statistical and computer skills) and also a broad knowledge of the underlying biology. When planning a PK/PD study, a question should be clearly raised by the intended user (generally a clinician having to design a clinical trial), keeping in mind that the generated results should be understandable in terms of scope and conclusions. For example, the question might be: what is the order of magnitude of the dose of a new analgesic to be tested in dogs in a clinical trial and what could be the clinical benefit of splitting the total daily dose into two doses at 12-h intervals? Conversely, for a given maximal dose of a timedependent antibiotic in pigs (fixed to take into account some environmental constraints), and knowing the MIC distributions of the main pathogens involved in pig pulmonary disease, what is the target attainment rate, that is, what is the percentage of a pig population able to maintain their plasma concentration for $40 \%$ of the dosing interval above the MIC for an empirical antibiotherapy (MIC a priori unknown)? A PK/PD study can also be planned to investigate some pharmacological properties of a drug such as the COX1 versus COX2 selectivity for a new coxib or to bridge some in vitrolex vivo results with the in vivo situation as is the case when using tissue cage fluid (exudate vs. transudate) to document the PD properties of antibiotics.

The selection of an experimental disease model (experimental inflammation to test an NSAID, experimental infection to test an antibiotic) or of an experimental challenge 
(painful stimulus to investigate an analgesic, histamine-induced cutaneous wheal formation to test an antihistaminic drug) should be critically scrutinized regarding the intended use of the drug. Experimental models are usually severe and the estimated parameter can lead to relatively high doses compared with what is actually needed in a clinical setting. Similarly, the selection of a biomarker, a surrogate marker, or a clinical end point should be carefully determined to provide data having appropriate metrological performance (sensitive, gradual, and reproducible) and being overall meaningful. For example, a preclinical PK/PD approach was successfully used to determine a dose of GnRH, for the treatment of ovarian follicular cysts in cattle using the pituitary LH response as a surrogate end point (Monnoyer et al., 2004). This is because the GnRH may produce an LH response similar to the preovulatory LH surge and may initiate estrus cycles in cows with ovarian follicle cysts. In contrast, using ACE inhibition to determine a dosage regimen for ACE inhibitors like benazepril, ramipril, or enalapril, is more delicate because the relationship between ACE inhibition and the prolongation of survival time or the prevention of kidney failure is not direct and straightforward. The measurement of a clinical end point, for example, the latency of paw withdrawal time elicited by a painful stimulus when testing an analgesic, or the measurement of lameness using force plates when assessing an NSAID, is by essence more meaningful than a biochemical biomarker of stress such as secretion of cortisol. Here, the difficulty is to guarantee objective and reproducible measurements and this requires a long-term training of animals enrolled in a trial and also of investigators. It is wise to have the same metrological validation for end point measurements in a PK/PD trial as for analytical techniques (accuracy, intra- and inter-day precision) even for easy measurements like body temperature. In addition, all measurements should be carried out in blinded conditions for the investigators; this practically requires crossover trials to be designed with both a placebo and a test article period. Despite all these precautionary measures, PD variables are often less precisely measured than the plasma concentration with a coefficient of variation of reproducibility reaching up to $30 \%$ (Botrel et al., 1994).

Owing to the inclusion of nonlinear steps in most PK/PD models, a sufficiently wide range of drug concentration is necessary to correctly estimate $E_{\max }$ and $E C_{50}$. Often several dose levels (including dose 0 or placebo) are tested and fitted together to cover the entire concentration-effect relationship. When a time-dependent baseline is included in the model to reflect some natural biorhythm (e.g., a circadian rhythm for cortisol when modeling the negative feedback of glucocorticoïds on cortisol secretion) or a reversible pathological process (e.g., an experimental inflammation when testing NSAID or the natural course of a disease when modeling a tolerance phenomenon), the time development of these processes in the absence of drug needs to be carefully assessed.

Developing a PK/PD model requires not only a general knowledge of modeling but also a biological understanding of the primary system under investigation. A collection of physiological models is given by the Physiome Project (http://nsr.bioeng.washington.edu/), which can be a good starting point to develop a suitable physiologically based PK/PD model.

Most PK/PD models have to be written with sets of differential equations because they generally include several nonlinear processes, and equations are solved numerically. Then parameters of the model (drug and system related) are estimated by nonlinear regression. Several computer programs exist to build defined user-friendly PK/PD models including 
Phoenix ${ }^{\circledR}$ WinNonlin ${ }^{\circledR}$ (Pharsight, Moutainview, CA), Kinetica (Innaphase, Philadelphia, PA), and Adapt II (Biomedical simulation resource, Los Angeles, CA). The modeling section of Chapter 14 should be consulted for additional details. To estimate parameters, initial values are needed. Vector of system parameters, that is, the component of the model that is independent of the tested drug, can be obtained from a priori knowledge. If system parameters are well known, they can be limited to the range of their possible physiological values (as for body temperature for a system model built to investigate the antipyretic action of NSAIDs or blood pressure for an antihypertensive drug). For drug-related parameters like $E C_{50}$ and $I C_{50}$, initial values can be obtained from in vitro knowledge of substance potency.

The final PK/PD model should be simple enough to remain identifiable with the available experimental data. Indeed, the goal is not to achieve the best fit of observed data but to provide useful information to the end users by making accurate and precise predictions. As the number of parameters in a model increases, the goodness of fit increases but at the expense of estimating the model parameters with an appropriate precision, so the risk is to have an overparameterized model.

When the goal is only to simulate situations (as in predictive toxicology), complicated physiologically based models (PBPK) can be developed and the parameters fixed to some a priori values issuing from what is rather well known (like blood flow, organ weights) or from in vitro investigations. Here, the challenge relies on the accuracy of the prediction: when the goal is to estimate system- and drug-related parameters, the question of structural and numerical identifiability should be addressed. Structural identifiability, a concept introduced in Chapter 8, refers to the uniqueness of estimating the model's parameters given model and error-free data. Numerical identifiability (or estimability) refers to the possibility of accurately estimating parameters of a structurally identifiable model given real, observed data (Bonate, 2006). A convenient approach to test identifiability is to simulate data sets and then fit the model to the error-free simulated data using the parameters used to simulate the data as starting values. If a model is identifiable, convergence is achieved within a few iterations, the residual sum of the squares is very small, and the precision of estimates is excellent (small standard error). If not identifiable, the model may not converge or, if it converges, the parameters would have large standard errors despite the model having a low residual sum of squares. If the model is not identifiable, many combinations of parameter estimates can be obtained. The solution to this problem can be (1) re-parametrization of the model especially to solve problems of estimability; (2) further simplification of the model by combining several parameters; or (3) conversely keeping the model, but collecting more information to render observable every parameter (i.e., to be sure that any parameter has an influence on the collected data). Distinguishability is another similar issue where different structural models can produce exactly the same output profile as the one that was observed.

After having fitted data, the model is generally used to simulate scenarios such as the influence of different dosage regimens on the response. Mean or median parameter values obtained from a pool of individuals (two stages analysis) or typical values as given by a population modeling approach are selected to run the model. With a population model, estimates of inter- and intra-animal variability enable population data to be generated. This is of special interest for PK/PD modeling of antibiotics, where one of the objectives is to achieve a target attainment rate in a given percentile of the population. Finally, simulations are also the best way to communicate with end users. 


\subsubsection{Application of PK/PD concepts to in vitro/in vivo extrapolations}

Extrapolation from in vitro to in vivo is another fruitful application of the PK/PD paradigm. Quantitative exposure-response relationships are often easily obtained from some in vitro or ex vivo system, for example, MIC for antibiotics, whole blood assay for NSAIDs, intact pathogenic organism in culture for anthelmintic drug discovery, and toxicological responses in tissue culture. If an effective concentration ( $E C$ for stimulation, $I C$ for inhibition) is obtained on the basis of an in vitro or ex vivo assay, then a dose can be proposed by incorporating the in vitro EC directly into Equation 13.2. It should be noted that since in vitro concentrations are generally equivalent to free drug concentrations, corrections for drug binding to plasma proteins might be needed to estimate the corresponding in vivo plasma $E C$ or $I C$. Chapter 5 discussed methods to accomplish this.

\subsection{APPLICATION OF THE PK/PD APPROACH TO THE SELECTION OF AN EFFECTIVE DOSAGE REGIMEN}

\subsubsection{The case of NSAIDs}

The main application of a PK/PD investigation is to document or suggest a dosage regimen for pivotal clinical trials. An initial approach consists of computing an average daily dose from Equation 13.2 using estimated PD parameters. For example, using Freund's adjuvant model in the dog, the $I C_{50}$ of nimesulide for lameness was found to be $6.26 \mu \mathrm{g} / \mathrm{mL}$ (Toutain et al., 2001b). Using Equation 13.2 and considering the plasma clearance of nimesulide $\left(15.3 \mathrm{~mL} / \mathrm{kg} / \mathrm{h}\right.$ or $367.2 \mathrm{~mL} / \mathrm{kg} /$ day) and its oral bioavailability $(47 \%)$, the $E D_{50}$ of oral nimesulide administration for the treatment of lameness can be calculated as $4.9 \mathrm{mg} / \mathrm{kg} /$ day. This is nearly equal to the recommended dose $(5 \mathrm{mg} / \mathrm{kg})$. A more advanced approach consists of simulating a previously established PK/PD model in order to allow the clinician to inspect the time course of the effect obtained at different dose levels in order to assist him or her in selecting a dose and a dosing interval for confirmatory clinical trials. For this, the relationship between plasma NSAID concentrations and a relevant clinical outcome such as body temperature for fever or a lameness score for locomotive inflammation needs to be incorporated. Different possible dosage regimens (dose, interval of administration, modalities of administration) can then be simulated as illustrated in Fig. 13.20 for a hypothetical NSAID.

The second parameter to be determined in a rational multiple-dose regimen is the time interval between administrations. Using a PK/PD model, a large number of dose and dosage interval scenarios can be simulated to select a dosage regimen having the best efficacy or safety margins. Such analysis requires no additional time or cost during drug development. For example, as seen in Fig. 13.21, it was shown that a PK/PD model predicted a better antipyretic efficacy for nimesulide at a dosage regimen of $2.5 \mathrm{mg} / \mathrm{kg}$ twice a day rather than at $5 \mathrm{mg} / \mathrm{kg}$ once per day, although both dosage regimens were equivalent in terms of lameness suppression.

Another aspect of PK/PD modeling is to document drug selectivity. Here, one of the most convenient approaches consists of documenting the differential action of the tested NSAID on COX1 and COX2 inhibition using an ex vivo experimental model of acute inflammation involving surgically implanted tissue cages (Lees et al., 2004). Then the ratio 


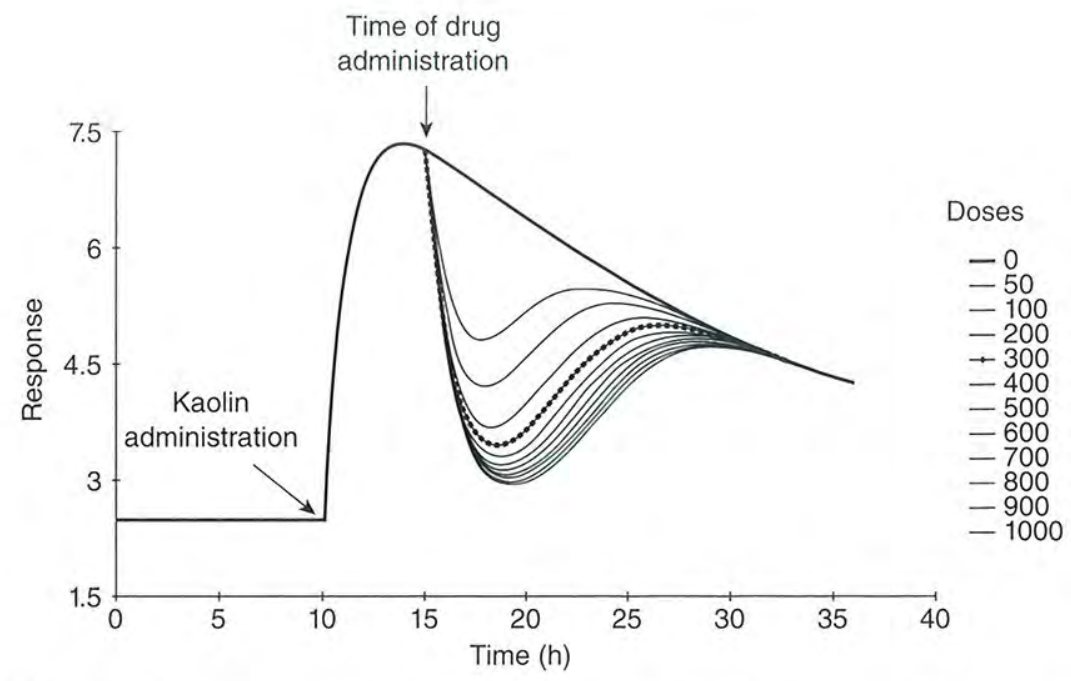

Fig. 13.20 Dose-effect relationship for a hypothetical NSAID on the reduction of lameness in experimental paw inflammation. An indirect effect model was used to simulate data. Parameters of the model were obtained from a previous experiment in which a phlogogenic agent (kaolin) was administered to create an experimental inflammation of the paw at $10 \mathrm{~h}$. The drug was then administered at $15 \mathrm{~h}$ and the degree of lameness assessed before kaolin administration (negative control), after kaolin administration but before drug administration (positive control), and then after the NSAID administration. The experimental data were modeled with the following model:

$$
d R / d t=K_{\text {in }}+\text { Inflammation } \cdot\left(1-\frac{[\text { Drug }]}{\left[E C_{50}+\text { Drug }\right]}\right)-K_{\text {out }} \cdot R
$$

where $R$ is the lameness response versus time (h), and Inflammation is the function describing the time course of the kaolin effect on locomotion in the absence of drug. In this example, inflammation was modeled with Equation 13.20, Inflammation was mitigated by the NSAID through a fractional inhibitory model with Drug, the NSAID plasma concentration as given by the pharmacokinetic model describing the NSAID disposition (here a monocompartmental model with a phase of absorption) and $E C_{50}$ is the NSAID plasma concentration for which the inhibitory function is at $50 \%$ of its maximum capacity. Then, using this model with the different estimated parameters, the time course of the test drug effect was simulated for doses ranging from 0 (placebo) to 1000. Visual inspection of the different curves shows that the effect increased progressively with the dose but not proportionally. With higher doses, there is a "diminishing return" because the incremental increase in effect is smaller with each incremental increase in the NSAID dose, and beyond the dose of $300(-\bullet-)$, the increase of the maximal effect becomes marginal and only the duration of effect is slightly prolonged. Therefore, it is likely that a clinician will consider the 300 dose to be a putative dose for testing in a clinical trial.

$E C_{50, \mathrm{COXX}_{2}} / E C_{50, \mathrm{COX} 1}$ can be considered as an index to predict in vivo selectivity. However, it should be borne in mind that the selected end points are surrogates and not actual outcomes of direct clinical interest.

\subsubsection{The case of antibiotics}

PK/PD modeling was first developed for reversibly acting drugs, but it can also successfully be applied to irreversible drug effects as is the case for antibiotics. For example, the 


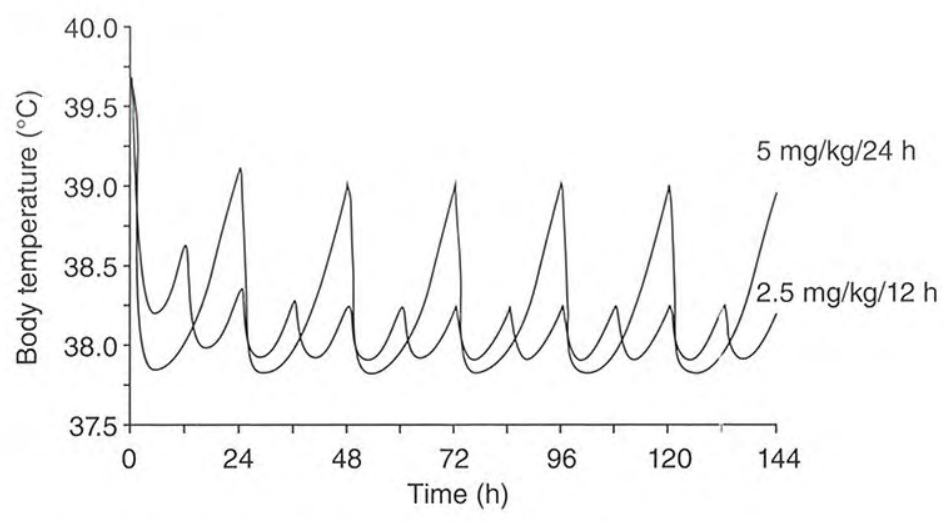

Fig. 13.21 Plot of predicted body temperature $\left({ }^{\circ} \mathrm{C}\right)$ versus time $(\mathrm{h})$ afier administration of nimesulide in dogs at two different doses $(2.5 \mathrm{mg} / \mathrm{kg} / 12 \mathrm{~h}$ and $5 \mathrm{mg} / \mathrm{kg} / 24 \mathrm{~h}$, for 6 consecutive days). Visual inspection of the figure suggests the superiority of the $2.5 \mathrm{mg} / \mathrm{kg} / 12 \mathrm{~h}$ dosage regimen (from Toutain et al., 2001a).

Hill equation can be used to describe the activity of an antibiotic against microorganisms as follows:

$$
d N / d t=B \cdot\left(K_{\text {growth }}-\frac{K_{\text {kill_max }} \cdot C(\hat{t})^{n}}{E C_{50}^{n}+C(t)^{n}}\right)
$$

where $B$ is the bacterial population size (colony-forming units/milliliter), $K_{\text {growth }}$ is the apparent rate constant of exponential growth without exposure to the antibiotic (unit: $1 /$ time), $K_{\text {kill_max }}(1 /$ time $)$ is the maximum bactericidal effect, $n$ is the Hill coefficient of sigmoidicity, $C(t)$ is the antibiotic plasma concentration at time $t$, and $E C_{50}$ is the antibiotic concentration producing $50 \%$ of $E_{\max }$. From this basic model first proposed by Zhi et al. (1986), many refinements have been proposed to describe situations where growth is limited by the shortage of resources, emergence and replication of resistant subpopulations, action of antibiotics either on the replication rate or on the killing rate, and influence of immune response. Using these kinds of mechanistic models, incorporating several key factors contributing to the emergence of resistance has allowed different modalities of antibiotic administration to be simulated. For example, it was shown that the early initiation of treatment and combination therapy with two antibiotics prevented the emergence of resistant bacteria, whereas a shorter course of therapy and sequential administration of antibiotics promoted the emergence of resistance (D'Agata et al., 2008).

These PK/PD models are able to predict some general features of antibiotic/pathogen interaction. Most of the time, the so-called time-dependent antibiotics such as beta-lactams have a high Hill coefficient and a low $E_{\max }$ (low maximum killing rate) while the so-called concentration-dependent antibiotics such as quinolones and aminoglycosides are often characterized by a low Hill coefficient and a high $E_{\max }$, meaning that the same Hill equation is able to describe the action of the different antibiotic classes. 
The lack of sensitivity of clinical outcomes to determine the best dosage regimen in terms of bacteriological cure opens the way for investigating the efficacy of antibiotics using surrogate PK/PD indices. Using the murine thigh and lung infection models, various empirical PK/PD indices have been proposed to predict the success or failure of a therapy. Three appear to be sufficient to predict antibiotic effectiveness: (1) the AUC/MIC ratio, an index used for quinolones; (2) the $C_{\max } / \mathrm{MIC}$ ratio (where $C_{\max }$ is the maximum plasma concentration), an index selected for aminoglycosides; and (3) $T>$ MIC (the time during which plasma concentrations exceed MIC, expressed as a percentage of the dosage interval), an index selected for the so-called time-dependent antibiotics such as beta-lactams. These were originally discussed as targets for constructing dosage regimens in Chapter 12 .

The parameter ratios AUC/MIC, $T>\mathrm{MIC}$, and $C_{\max } / \mathrm{MIC}$ are said to be PK/PD indices of efficacy because they combine a PK (AUC, $T>$ MIC, $C_{\max }$ ) and a common PD (MIC) parameter. These indices can be viewed as dose surrogates in a dose-titration setting (Fig. 13.2), the dose being replaced by a biomarker of exposure scaled by the MIC. Hence, they allow dual dosage individualization, that is, based on both the microbiological susceptibility and drug disposition kinetics. It should be noted that these PK/PD predictive indices of in vivo efficacy are again based on free, nonprotein-bound plasma concentrations of the antibiotic, not total tissue antibiotic levels.

A main goal of veterinary pharmacology is to determine breakpoint values for these three main PK/PD indices for each animal species and their pathogens. It can be done by assessing the ex vivo bacterial activity of an antibiotic in serum and tissue cage fluids (exudate, transudate) (e.g., Aliabadi and Lees, 2001). In this kind of experiment, the graded antibiotic response (expressed in terms of reduction of an initial bacterial count) is regressed against the surrogate marker (ex vivo $\mathrm{AUC}_{24 \mathrm{~h}} / \mathrm{MIC}$ ) using the Hill equation:

$$
\text { Antibacterial ex vivo response }=\frac{[\text { maximal possible drug effect }] \cdot(\text { surrogate })^{n}}{(\text { surrogate })_{50}^{n}+(\text { surrogate })^{n}}
$$

where the antibacterial response is measured in terms of reduction of the original bacterial count. The independent variable is the surrogate (e.g., $\mathrm{AUC}_{24 \mathrm{~h}} / \mathrm{MIC}$ ) for which a breakpoint is to be established. From Equation 13.35, it is possible to derive three parameters of clinical relevance: the maximal possible antibiotic effect (total bacterial eradication); the (surrogate $_{50}$, the value of the surrogate associated with $50 \%$ of the maximal effect and which is equivalent to an $E C_{50}$, that is, a measure of antibiotic potency; and $n$, the Hill coefficient, which gives the slope of the concentration-effect relationship. By solving the model, different levels of the antibacterial response (bacteriostasis, bactericidal effect) can be calculated and corresponding breakpoint values for the surrogate can be computed. Another option to determine the breakpoint value of PK/PD indices is to consider the relationship between the PK/PD index and the likelihood of clinical cure, that is, a quantal response using a logistic model as described by Equation 13.14 and illustrated in Fig. 13.22.

\subsection{CONCLUSIONS}

This chapter provided approaches to link the concentration-time profiles described by pharmacokinetic models to biological effect. The statistical approaches used to determine PD model parameter values are the same as those used in PK models fully addressed in the next chapter. 
Biomarker (log cfu/mL): graded responde (intensity of effect)

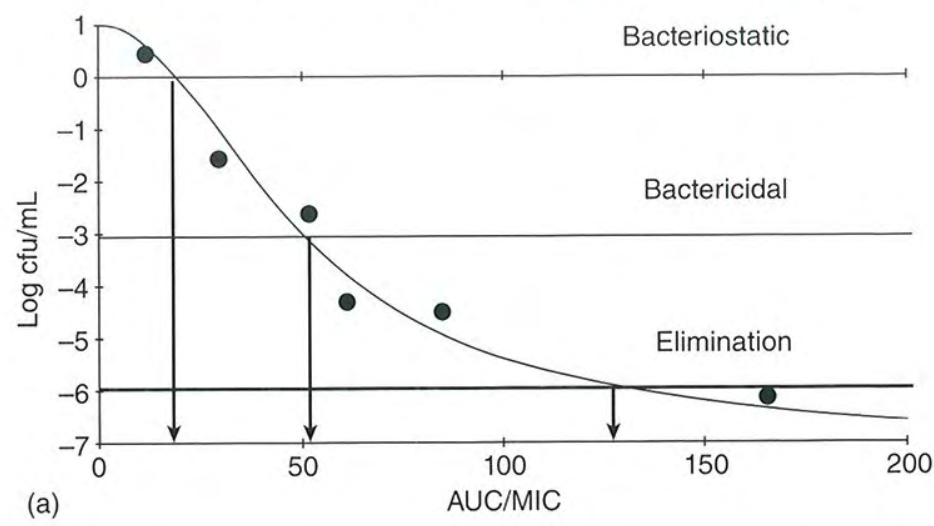

Clinical endpoint (cure rate \%); quantal response (frequency of response)

(b)

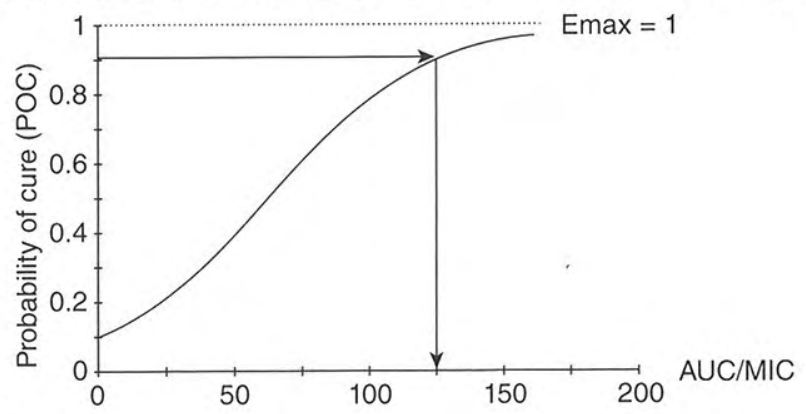

Fig. 13.22 Determination of a PK/PD breakpoint value for a quinolone (AUC/MIC). (a) Ex vivo approach consisting of measuring the reduction of an initial bacterial count (expressed as colony forming units [cfu] to different exposures of an antibiotic. Antibiotic exposure is scaled by the MIC of the tested pathogen allowing the AUC/MIC against the bacterial count to be regressed. From the fitted curve, the AUC/MIC corresponding to a bacteriostatic effect (no bacterial growth), bactericidal action (reduction by 3 log of the initial bacterial count) and eradication (reduction by about 6 log of the initial count) can be estimated (see also Fig. 13.10). (b) In vivo approach consisting of establishing a relationship between the probability of animal clinical cure and the observed AUC/MIC.

\section{BIBLIOGRAPHY}

Aliabadi, F.S., and Lees, P. 2001. Pharmacokinetics and pharmacodynamics of danofloxacin in serum and tissue fluids of goats following intravenous and intramuscular administration. American Journal of Veterinary Research. 62:1979-1989.

Aliabadi, F.S., and Lees, P. 2002. Pharmacokinetics and pharmacokinetic/pharmacodynamic integration of marbofloxacin in calf serum, exudate and transudate. Journal of Veterinary Pharmacology and Therapeutics. 25:161-174.

Atkinson, A.J., Colburn, W.A., DeGruttola, V.G., DeMets, D.L., Downing, G.J., Hoth, D.F., Oates, J.A., Peck, C.C., Schooley, R.T., Spilker, B.A., Woodcock, J., and Zeger, S.L. 2001. Biomarkers and surrogate endpoints: preferred definitions and conceptual framework. Clinical Pharmacology and Therapeutics. 69:89-95.

Bonate, P.L. 2006. Pharmacokinetic-Pharmacodynamic Modeling and Simulation. New York: Springer. 
Botrel, M.A., Haak, T., Legrand, C., Concordet, D., Chevalier, R., and Toutain, P.L. 1994. Quantitative evaluation of an experimental inflammation induced with Freund's complete adjuvant in dogs. Journal of Pharmacology and Toxicology Methods. 32:63-71.

Czock, D., and Keller, F. 2007. Mechanism-based pharmacokinetic-pharmacodynamic modeling of antimicrobial drug effects. Journal of Pharmacokinetics and Pharmacodynamics. 34:727-751.

D'Agata, E.M., Dupont-Rouzeyrol, M., Magal, P., Olivier, D., and Ruan, S. 2008. The impact of different antibiotic regimens on the emergence of antimicrobial-resistant bacteria. PLOS ONE. 3:e4036.

Dahl, S.G., Aarons, L., Gundert-Remy, U., Karlsson, M.O., Schneider, Y.J., Steimer, J.L., and Troconiz, I.F. 2009. Incorporating physiological and biochemical mechanisms into pharmacokineticpharmacodynamic models: a conceptual framework. Basic Clinical Pharmacology and Toxicology.
106:2-12.

Danhof, M., de Lange, E.C., Della Pasqua, O.E., Ploeger, B.A., and Voskuyl, R.A. 2008. Mechanism-based pharmacokinetic-pharmacodynamic (PK-PD) modeling in translational drug research. Trends in
Pharmacological Sciences. 29:186-191.

Dayneka, N.L., Garg, V., and Jusko, W.J. 1993. Comparison of four basic models of indirect pharmacodynamic responses. Journal of Pharmacokinetics and Biopharmaceutics. 21:457-478.

Ette, E.I., Roy, A., and Nandy, P. 2007. Population pharmacokinetic/pharmacodynamic modeling of ordered categorical longitudinal data. In: Ette, E.I., and Williams, P.J. (eds.), Pharmacometrics: The Science of Quantitative Pharmacology. Hoboken, NJ: John Wiley \& Sons.

Fiedler-Kelly, B. 2007. PK/PD analysis of binary (logistic) outcome data. In: Ette, E.I., and Williams, P.J. (eds.), Pharmacometrics: The Science of Quantitative Pharmacology. Hoboken, NJ: John Wiley \&
Sons.

Fosse, T.K., Toutain, P.L., Spadavecchia, C., Haga, H.A., Horsberg, T.E., and Ranheim, B. 2010. Ketoprofen in piglets: enantioselective pharmacokinetics, pharmacodynamics and PK/PD modelling. Journal of
Veterinary Pharmacology and Therapeutics (in press). Frank, R., and Hargreaves, R. 2003. Clinical biomarkers in drug discovery and development. Nature
Reviews Drug Discovery. 2:566-580.

Friedman, P.A., and Hebert, S.C. 1997. Site and mechanism of diuretic action. In: Seldin, D., and Giebisch, G. (eds.), Diuretic Agents. Clinical Physiology and Pharmacology. San Diego, CA: Academic Press.

Gabrielsson, J., and Weiner, D. 2006. Pharmacokinetic and Pharmacodynamic Data Analysis: Concepts and Applications. Stockholm, Sweden: Swedish Pharmaceutical Press.

Gardmark, M., Brynne, L., Hammarlund-Udenaes, M., and Karlsson, M.O. 1999. Interchangeability and predictive performance of empirical tolerance models. Clinical Pharmacokinetics. 36:145-167. Girard, P. 2005. Clinical trial simulation: a tool for understanding study failures and preventing them. Basic
and Clinical Pharmacology and Toxicology. 96:228-234. Girard, P., and Boissel, J.P. 1989. Clockwise hysteresis or proteresis. Journal of Pharmacokinetics and
Biopharmaceutics. 17:401-402.

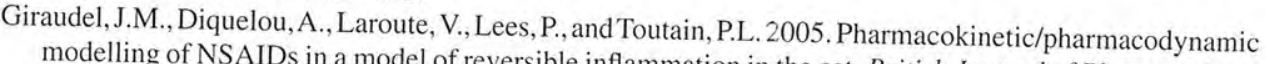
modelling of NSAIDs in a model of reversible inflammation in the cat. British Journal of Pharmacology.
146:642-653. Holford, N.H., and Sheiner, L.B. 1982. Kinetics of pharmacologic response. Pharmacology and
Therapeutics. 16:143-166.

Holford, N.H.G., and Ludden, T.M. 1994. Time course of drug effect. In: Welling, P.G., and Balant, L.P. (eds.), Pharmacokinetics of Drugs. Berlin: Springer-Verlag.

Jonker, D.M., Visser, S.A., van der Graaf, P.H., Voskuyl, R.A., and Danhof, M. 2005. Towards a mechanism-
based analysis of pharmacodynamic drug-drug based analysis of pharmacodynamic drug-drug interactions in vivo. Pharmacology and Therapeutics.
106:1-18. Jusko, W.J., and Ko, H.C. 1994. Physiologic indirect response models characterize diverse types of phar-
macodynamic effects. Clinical Pharmacology and Therapeutics. 56:406-419.

King, J.N., Maurer, M., and Toutain, P.L. 2003. Pharmacokinetic/ pharmacodynamic modelling of the
disposition and effect of benazepril and benazeprilat disposition and effect of benazepril and benazeprilat in cats. Journal of Veterinary Pharmacology and
Therapeutics. 26:213-224.

KuKanich, B., and Papich, M.G. 2009. Opioid analgesic drugs. In: Riviere, J.E., and Papich, M.G. (eds.), Veterinary Pharmacology \& Therapeutics, 9th Ed. Ames, IA: Wiley-Blackwell, pp. 301-336.

Lees, P., Giraudel, J., Landoni, M.F., and Toutain, P.L. 2004. PK-PD integration and PK-PD modelling of nonsteroidal anti-inflammatory drugs: principles and applications in veterinary pharmacology. Journal of Veterinary Pharmacology and Therapeutics. 27:491-502. 
Levy, G. 1964. Relationship between rate of elimination of tubocurarine and rate of decline of its pharmacological activity. British Journal of Anaesthesiology. 36:694-695.

Levy, G. 1998. Impact of pharmacodynamic variability on drug delivery (1). Advanced Drug Delivery Reviews. 33:201-206.

Mager, D.E., Wyska, E., and Jusko, W.J. 2003. Diversity of mechanism-based pharmacodynamic models. Drug Metabolism and Disposition. 31:510-518.

Meibohm, B., and Derendorf, H. 2002. Pharmacokinetic/pharmacodynamic studies in drug product development. Journal of Pharmaceutical Sciences. 91:18-31.

Monnoyer, S., Guyonnet, J., and Toutain, P.L. 2004. A preclinical pharmacokinetic/pharmacodynamic approach to determine a dose of $\mathrm{GnRH}$, for treatment of ovarian follicular cyst in cattle. Journal of Veterinary Pharmacology and Therapeutics. 27:527-535.

Mould, D.R. 2007. Developing models of disease progression. In: Ette, E.I., and Williams, P.J. (eds.), Pharmacometrics: The Science of Quantitative Pharmacology. Hoboken, NJ: John Wiley \& Sons.

Pillai, G., Gieschke, R., Goggin, T., Jacqmin, P., Schimmer, R.C., and Steimer, J.L. 2004. A semimechanistic and mechanistic population PK-PD model for biomarker response to ibandronate, a new bisphosphonate for the treatment of osteoporosis. British Journal of Clinical Pharmacology. 58:618-631.

Post, T.M., Freijer, J.I., DeJongh, J., and Danhof, M. 2005. Disease system analysis: basic disease progression models in degenerative disease. Pharmaceutical Research. 22:1038-1049.

Qiao, G.L., and Fung, K.F.. 1993. Pharmacokinetic-pharmacodynamic modelling of meperidine in goats (I): pharmacokinetics. Journal of Veterinary Pharmacology and Therapeutics. 16:426-437.

Sheiner, L.B., Stanski, D.R., Vozeh, S., Miller, R.D., and Ham, J. 1979. Simultaneous modeling of pharmacokinetics and pharmacodynamics: application to d-tubocurarine. Clinical Pharmacology and Therapeutics. 25:358-371.

Toutain, P.L., and Cester, C.C. 2004. Pharmacokinetic-pharmacodynamic relationships and dose response to meloxicam in horses with induced arthritis in the right carpal joint. American Journal of Veterinary
Research. 65:1533-1541.

Toutain, P.L., and Lees, P. 2004. Integration and modelling of pharmacokinetic and pharmacodynamic data to optimize dosage regimens in veterinary medicine. Journal of Veterinary Pharmacology and Therapeutics. 27:467-477.

Toutain, P.L., Autefage, A., Legrand, C., and Alvinerie, M. 1994. Plasma concentrations and therapeutic efficacy of phenylbutazone and flunixin meglumine in the horse: pharmacokinetic/pharmacodynamic modelling. Journal of Veterinary Pharmacology and Therapeutics. 17:459-469.

Toutain, P.L., Lefebvre, H.P., and King, J.N. 2000. Benazeprilat disposition and effect in dogs revisited with a pharmacokinetic/pharmacodynamic modeling approach. Journal of Pharmacology and Experimental Therapeutics. 292:1087-1093.

Toutain, P.L., Cester, C.C., Haak, T., and Laroute, V. 2001a. A pharmacokinetic/pharmacodynamic approach vs. a dose titration for the determination of a dosage regimen: the case of nimesulide, a Cox-2 selective nonsteroidal anti-inflammatory drug in the dog. Journal of Veterinary Pharmacology and Therapeutics. 24:43-55.

Toutain, P.L., Cester, C.C., Haak, T., and Metge, S. 2001b. Pharmacokinetic profile and in vitro selective cyclooxygenase- 2 inhibition by nimesulide in the dog. Journal of Veterinary Pharmacology and Therapeutics. 24:35-42.

Williams, P.J., and Ette, E.I. 2007. Biomarkers in drug development and pharmacometric modeling. In: Ette, E.I., and Williams, P.J. (eds.), Pharmacometrics: The Science of Quantitative Pharmacology. Hoboken, NJ: John Wiley \& Sons.

Zhi, J., Nightingale, C.H., and Quintiliani, R. 1986. A pharmacodynamic model for the activity of antibiotics against microorganisms under nonsaturable conditions. Journal of Pharmaceutical Sciences.
$75: 1063-1067$. 
Comparative Pharmacokinetics

Principles, Techniques, and Applications

Second Edition

Jim E. Riviere 
This edition first published 2011 (C) 2011 by Jim E. Riviere

First edition published 1999 (C) 1999 Iowa State University Press

Blackwell Publishing was acquired by John Wiley \& Sons in February 2007. Blackwell's publishing program has been merged with Wiley's global Scientific, Technical and Medical business to form Wiley-Blackwell.

Registered office: John Wiley \& Sons, Ltd, The Atrium, Southern Gate, Chichester, West Sussex, PO19 8SQ, UK

Editorial offices: 2121 State Avenue, Ames, Iowa 50014-8300, USA

The Atrium, Southern Gate, Chichester, West Sussex, PO19 8SQ, UK

9600 Garsington Road, Oxford, OX4 2DQ, UK

For details of our global editorial offices, for customer services and for information about how to apply for permission to reuse the copyright material in this book please see our website at www.wiley.com/

wiley-blackwell.

Authorization to photocopy items for internal or personal use, or the internal or personal use of specific clients, is granted by Blackwell Publishing, provided that the base fee is paid directly to the Copyright Clearance Center, 222 Rosewood Drive, Danvers, MA 01923. For those organizations that have been granted a photocopy license by CCC, a separate system of payments has been arranged. The fee codes for users of the Transactional Reporting Service are ISBN-13: 978-0-8138-2993-7/2011.

Designations used by companies to distinguish their products are often claimed as trademarks. All brand names and product names used in this book are trade names, service marks, trademarks or registered trademarks of their respective owners. The publisher is not associated with any product or vendor mentioned in this book. This publication is designed to provide accurate and authoritative information in regard to the subject matter covered. It is sold on the understanding that the publisher is not engaged in rendering professional services. If professional advice or other expert assistance is required, the services of a competent professional should be sought.

\section{Library of Congress Cataloging-in-Publication Data}

Riviere, Jim E. (Jim Edmond), author.

Comparative pharmacokinetics / Jim E. Riviere, North Carolina State University, Raleigh, North Carolina. - 2nd Edition.

p. ; cm.

Includes bibliographical references and index.

ISBN 978-0-8138-2993-7 (pbk. : alk. paper)

1. Pharmacokinetics. 2. Veterinary pharmacology. I. Title.

[DNLM: 1. Pharmacokinetics. QV 38]

RM301.5.R58 2011

$636.089^{\prime} 578-\mathrm{dc} 22$

2010047729

A catalogue record for this book is available from the British Library.

Set in 10/12pt Times by Toppan Best-set Premedia Limited

\section{Disclaimer}

The publisher and the author make no representations or warranties with respect to the accuracy or completeness of the contents of this work and specifically disclaim all warranties, including without limitation warranties of fitness for a particular purpose. No warranty may be created or extended by sales or promotional materials. The advice and strategies contained herein may not be suitable for every situation. This work is sold with the understanding that the publisher is not engaged in rendering legal, accounting, or other professional services. If professional assistance is required, the services of a competent professional person should be sought. Neither the publisher nor the author shall be liable for damages arising herefrom. The fact that an organization or Website is referred to in this work as a citation and/or a potential source of further information does not mean that the author or the publisher endorses the information the organization or Website may provide or recommendations it may make. Further, readers should be aware that Internet Websites listed in this work may have changed or disappeared between when this work was written and when it is read. 